\title{
Comparison of Geoacoustic Models for Unfrozen Water Content Estimation
}

Lyu, Chuangxin; Amiri, Seyed Ali Ghoreishian; Grimstad, Gustav; Høyland, Knut Vilhelm; Ingeman Nielsen, Thomas

Published in:

Journal of Geophysical Research: Solid Earth

Link to article, DOI:

10.1029/2020JB019766

Publication date:

2020

Document Version

Publisher's PDF, also known as Version of record

Link back to DTU Orbit

Citation (APA):

Lyu, C., Amiri, S. A. G., Grimstad, G., Høyland, K. V., \& IngemanNielsen, T. (2020). Comparison of Geoacoustic Models for Unfrozen Water Content Estimation. Journal of Geophysical Research: Solid Earth, 125(10), [e2020JB019766]. https://doi.org/10.1029/2020JB019766

\section{General rights}

Copyright and moral rights for the publications made accessible in the public portal are retained by the authors and/or other copyright owners and it is a condition of accessing publications that users recognise and abide by the legal requirements associated with these rights.

- Users may download and print one copy of any publication from the public portal for the purpose of private study or research.

- You may not further distribute the material or use it for any profit-making activity or commercial gain

- You may freely distribute the URL identifying the publication in the public portal 


\section{JGR Solid Earth}

\section{RESEARCH ARTICLE \\ 10.1029/2020JB019766 \\ Comparison of Geoacoustic Models for Unfrozen Water Content Estimation}

Special Section:

The Arctic: An AGU Joint

Special Collection

Key Points:

- Review of combined laboratory data shows that geoacoustic models can estimate the unfrozen water content for saline frozen soils

- Joint analysis of data and models indicates the dependency of ice crystallization mode on the unbound water content and salinity of the soil

- Salt precipitation and dissolution are interpreted as the likely cause of freeze-thaw hysteresis near the eutectic temperature in frozen soil

Correspondence to:

C. Lyu,

chuangxin.lyu@ntnu.no

Citation:

Lyu, C., Amiri, S. A. G., Grimstad, G., Høyland, K. V., \& Ingeman-Nielsen, T. (2020). Comparison of geoacoustic models for unfrozen water content estimation. Journal of Geophysical Research: Solid Earth, 125, e2020JB019766. https://doi.org/ 10.1029/2020JB019766

Received 16 MAR 2020 Accepted 17 SEP 2020 Accepted article online 20 SEP 2020

(C)2020. American Geophysical Union. All Rights Reserved.

\author{
Chuangxin Lyu ${ }^{1}$ (D), Seyed Ali Ghoreishian Amiri ${ }^{1}$, Gustav Grimstad ${ }^{1}$, \\ Knut Vilhelm Høyland ${ }^{1}$, and Thomas Ingeman-Nielsen ${ }^{2}$ (D) \\ ${ }^{1}$ Department of Civil and Environmental Engineering, Norwegian University of Science and Technology (NTNU), \\ Trondheim, Norway, ${ }^{2}$ Department of Civil Engineering, Technical University of Denmark (DTU), Kongens Lyngby, \\ Denmark
}

\begin{abstract}
This paper reviews eight geoacoustic models applied to frozen soils: crystal growth models (grain cementing, grain coating, matrix supporting, and pore filling), the weighted equation (WE) model, Zimmerman and King's model (KT), the Biot-Gassmann theory modified by Lee (BGTL), and a two-end member model. We verify the capacity of these models to estimate unfrozen water content (UWC) based on "reference" UWC results and joint $P$ and $S$ wave velocities for different soil types. The satisfactory UWC estimates of saline unconsolidated sand and overconsolidated clay based on $V_{p}$ data prove that the KT, BGTL, and two-end member models are capable of modeling "smooth" transitions in the ice crystal growth mode, while they may provide less accurate UWC values when abrupt change of crystallization mode occurs. None of the tested soil types show a single crystallization mode throughout the freezing process, as assumed by individual crystal growth models. $V_{s}$-based UWC estimates are less accurate due to significant but difficult-to-estimate influence of effective stress and soil initial cementation. All models, except pore filling and matrix supporting, can match $V_{s}$ versus $V_{p}$ measurement results for sands and silts but gradually provide inconsistent estimates with increasing clay content. We conclude that model validation by independent UWC measurements is necessary and that consistency between UWC values estimated from $V_{s}$ and $V_{p}$ is insufficient to ensure proper model validation.
\end{abstract}

\section{Introduction}

Saturated frozen soil is a multiphase porous material that consists of unfrozen water, ice, and solid (soil) particles. The determination of unfrozen water content (UWC) is important for permafrost engineering and permafrost-climate interaction studies (Humlum et al., 2003; Koven et al., 2011, 2013; Qingbai et al., 2002), since the thermo-hydro-mechanical behavior of frozen soils is highly dependent on the UWC. For instance, a change in UWC can vary the hydraulic conductivity of frozen soils up to several orders of magnitude and thus critically impacts the hydrological properties of frozen soil (Williams \& Burt, 1974). There are several available laboratory measurement methods for determination of the UWC, such as the calorimetric method (Dillon \& Andersland, 1966; Williams, 1964), nuclear magnetic resonance (NMR) (Anderson \& Tice, 1973; Kruse et al., 2018; Watanabe \& Wake, 2009), and time domain reflectometer (TDR) (Smith \& Tice, 1988). However, UWC determination at large-scale field investigation is still an issue.

Acoustic methods have often been used in permafrost investigations to determine the UWC. The method is nondestructive and relatively easy to implement (Hauck et al., 2007; Vonder Mühll et al., 2002). It is an indirect method, and the estimate accuracy depends on the reliability of the geoacoustic models. Several models are available, such as the weighted equation (WE) (Lee et al., 1996), the effective medium theories (Dou et al., 2017; Zimmerman \& King, 1986), and the extended three-phase Biot theory (Leclaire et al., 1994). However, these geoacoustic models have not been consistently validated against more accurate laboratory methods such as NMR and TDR. Indeed, limitation of the models and their applicability for different soil types are not sufficiently investigated.

This paper reviews available parametric studies of soil type, temperature, and salinity effects on acoustic $P$ and $S$ wave velocities of frozen soil and revisits their physical mechanisms (sections 2.1-2.3). Based on the review, we suggest two new hysteresis mechanisms: (1) trapped air-induced hysteresis in the process of freezing and (2) salt dissolution-precipitation hysteresis around eutectic temperatures (section 2.3). We go 

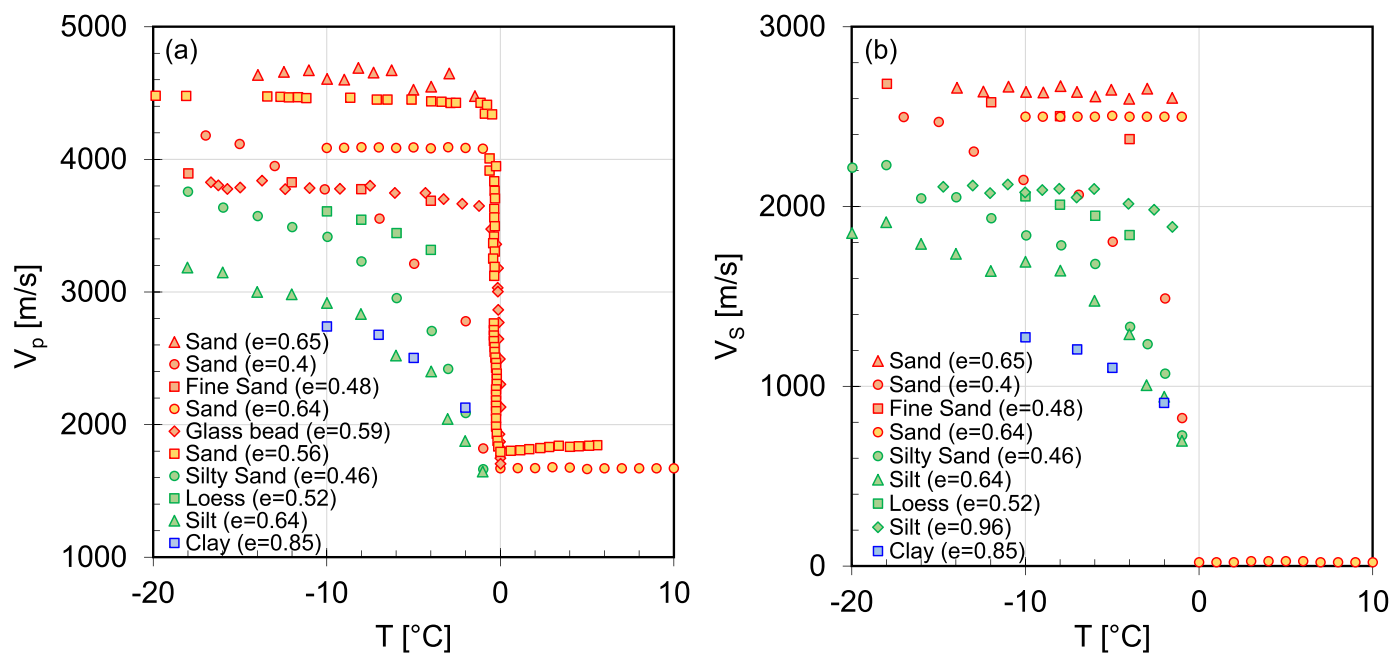

Figure 1. Effect of temperature and soil types on acoustic velocities for unconsolidated permafrost. (a) $P$ wave velocity $V_{p}$ versus temperature $T$; (b) $S$ wave velocity $V_{S}$ versus temperature $T$; $e$ corresponds to void ratio. Sand $(e=0.65)$ (Nakano \& Arnold, 1973); sand ( $e=0.4)$, silt sand $(e=0.46)$, and silt $(e=0.64)$ (Christ \& Park, 2009); fine sand $(e=0.48)$, loess $(e=0.52)$, and clay $(e=0.85)$ (Wang et al., 2006); sand ( $e=0.64)$ (Park \& Lee, 2014); glass bead $(e=0.59)$ (Deschatres et al., 1988); sand ( $e=0.56)$ (Dou et al., 2016); silt $(e=0.96)$ (Nakano et al., 1972).

on to review eight geoacoustic models, their physical basis, and previous application to frozen soils. The original contribution of the paper consists of the intercomparison of those geoacoustic models to consistent data sets of acoustic velocities as function of UWC (as measured by NMR or TDR) from published studies (section 4.3) and discussion of their performance with clear physical explanations (section 5).

\section{Parametric Analysis of Acoustic Velocity Data in Frozen Soils}

This section revisits previous acoustic measurements and the revealed mechanisms with regard to the effect of temperature, soil type, salinity, and freezing-thawing on the acoustic velocity in frozen soils.

\subsection{Acoustic Velocities Versus Soil Type and Temperature}

Figure 1 presents $P$ and $S$ wave velocity measurements as a function of temperature for different types of frozen soil, from sand to clay with low salinities. Both the $P$ and $S$ wave data show similar trends with increasing velocities for decreasing temperature. A sharp increase in velocities is observed at the freezing temperature (around $0^{\circ} \mathrm{C}$ ) for sand, after that both velocities slowly increase with continued freezing. However, fine-grained soils show a more gradual increase with decreasing temperature. In these cases, the capillary and intermolecular interactions dominate the freezing process and cause more gradual freezing with higher UWC at lower temperatures (Wettlaufer \& Worster, 2006). The increase in $P$ and $S$ wave velocity is still quite significant near the ice initiation point (freezing temperature). It indicates that the stiffness of unconsolidated permafrost is highly sensitive to ice crystal growth behavior at the start of freezing (Matsushima et al., 2016).

\subsection{Acoustic Velocity Versus Salinity}

Figure 2 presents the effect of salinity on $P$ wave velocity. The presence of dissolved salts results in a freezing point depression, with effective freezing point as low as approximately $-10^{\circ} \mathrm{C}$ at a salinity $S=146 \mathrm{~g} / \mathrm{L}$ for frozen sand (Dou et al., 2016) due to formed hydration shells around the solvated ions. This structure is more disordered and results in an increase in the enthalpy difference between the liquid and solid states of water. As a result, more energy needs to be removed during phase change (Dou et al., 2016; Santamarina et al., 2001). Besides, the eutectic point, that is, the lowest possible melting point of the mixture, is $-21.3^{\circ} \mathrm{C}$ for the $\mathrm{NaCl}-\mathrm{H}_{2} \mathrm{O}$ binary system. Below this temperature, all the dissolved $\mathrm{Na}^{+}$and $\mathrm{Cl}^{-}$ions will precipitate, and unbound water molecules will therefore freeze rapidly at the eutectic 

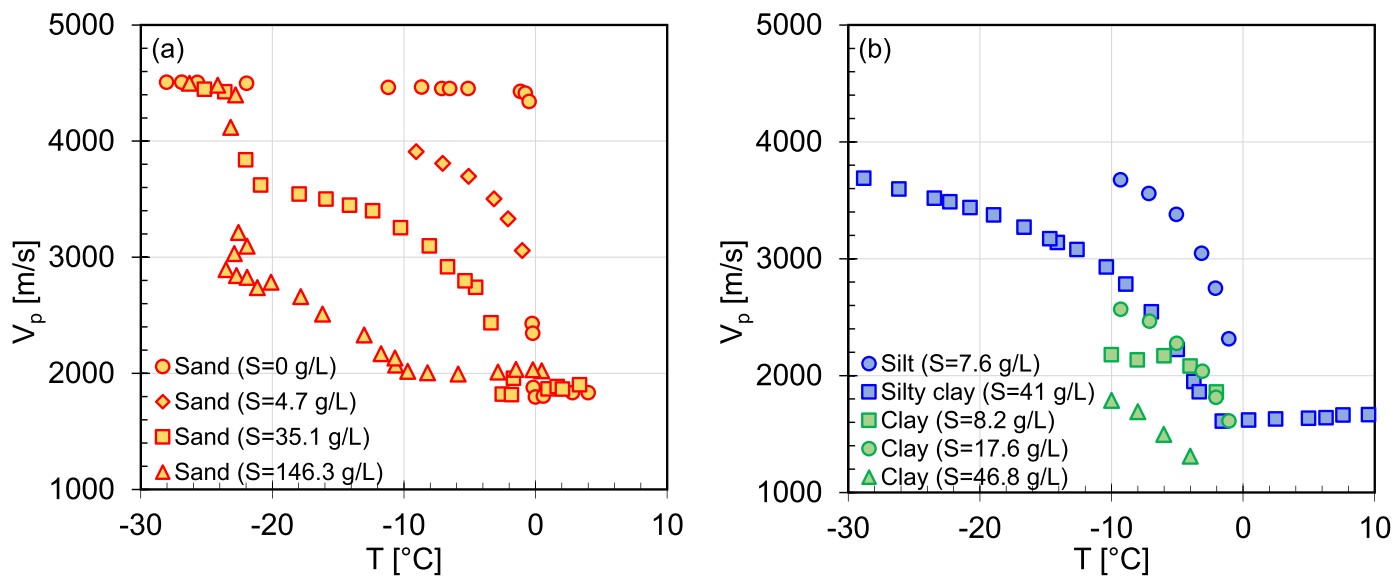

Figure 2. (a and b) Effect of salinity $S$ on $P$ wave velocity $V_{p}$ for unconsolidated permafrost. Sand $(S=0 \mathrm{~g} / \mathrm{L})$, sand $(S=35.1 \mathrm{~g} / \mathrm{L})$, sand $(S=146.3 \mathrm{~g} / \mathrm{L})$, silty clay $(S=41 \mathrm{~g} / \mathrm{L})$ (Dou et al., 2016); sand $(S=4.7 \mathrm{~g} / \mathrm{L})$, silt $(S=7.6 \mathrm{~g} / \mathrm{L})$, clay $(S=8.2 \mathrm{~g} / \mathrm{L})$, clay $(S=17.6 \mathrm{~g} / \mathrm{L})$, and clay $(S=46.8 \mathrm{~g} / \mathrm{L})$ (King et al., 1982).

temperature. As a result, a sharp increase in the soil stiffness at the eutectic point is observed in Figure 2 for saline sand (35.1 and $146.3 \mathrm{~g} / \mathrm{L}$ ) around the eutectic temperature of the $\mathrm{NaCl}$ solution as explained by Dou et al. (2016).

The increase in $P$ wave velocity versus temperature also becomes more gradual with increase in salinity as suggested in Figure 2. The electrolyte concentration of unfrozen water increases with ice crystallization, because the ions are expelled from the freezing ice to unfrozen water (King et al., 1988). The increase in salinity results in a further freezing point depression of the remaining unfrozen water. The ionic migration can also enhance the accumulation of positive and hydrated ions and raise concentration potential of bound water in the vicinity of negatively charged clay particles. This effect will generate sufficient osmotic potentials and adsorption force to yield the unfrozen bound water as temperature further declines. (Harrysson Drotz et al., 2009; Horiguchi, 1987; Ma et al., 2017; Torrance \& Schellekens, 2006). The latter mechanism for bound water is induced by surface charge and causes different effects of electrolytes in sand and clay soils. Therefore, the $P$ wave velocity of frozen saline clay is not expected to increase abruptly at the eutectic temperature (as is the case for frozen sand) since the bound water induced by surface effects of frozen clays will not freeze spontaneously.

\subsection{Acoustic Velocity Versus Freezing Thawing}

Several studies have presented evidences of temperature hysteresis in acoustic velocity (Dou et al., 2016; Nakano et al., 1972; Thimus et al., 1991). Figure 3 illustrates such a hysteresis behavior in laboratory measurements on different soil types at different salinities. Three different types of hysteresis are identified and described below, based on their distinctive dominating mechanisms (Wang et al., 2017, 2018).

Type I: supercooling-induced hysteresis. Supercooling is a metastable state for water molecules, which may occur when the water is cooled below its equilibrium freezing point. Supercooled water will freeze spontaneously when the temperature becomes low enough or when ice microparticles grow large enough to act as nucleation points (Dou et al., 2016). As this effect only influences the freezing process, the Type I hysteresis can be observed. Kozlowski (2009) speculates strong effects of soil plasticity and the sample mass on supercooling, and higher water content soils shows more significant supercooling hysteresis, which seems to be in line with the data presented in Figure 3. However, the question of the absence or presence for the supercooling of frozen soil remains open.

Type II: "ink-bottle" effect, ice lens growth, and trapped air-induced hysteresis. The ice phase invasion into water-filled pores is easier for large pores due to the capillary force difference, and ice in small pores is easier to transform into liquid phase because of the smaller enthalpy difference between the liquid and solid states. Therefore, freezing starts in the larger pores and progresses to the smaller pores, while thawing initiates from smaller pores. This is referred to as the "ink-bottle" effect (Dou et al., 2016). Pore size distribution and 

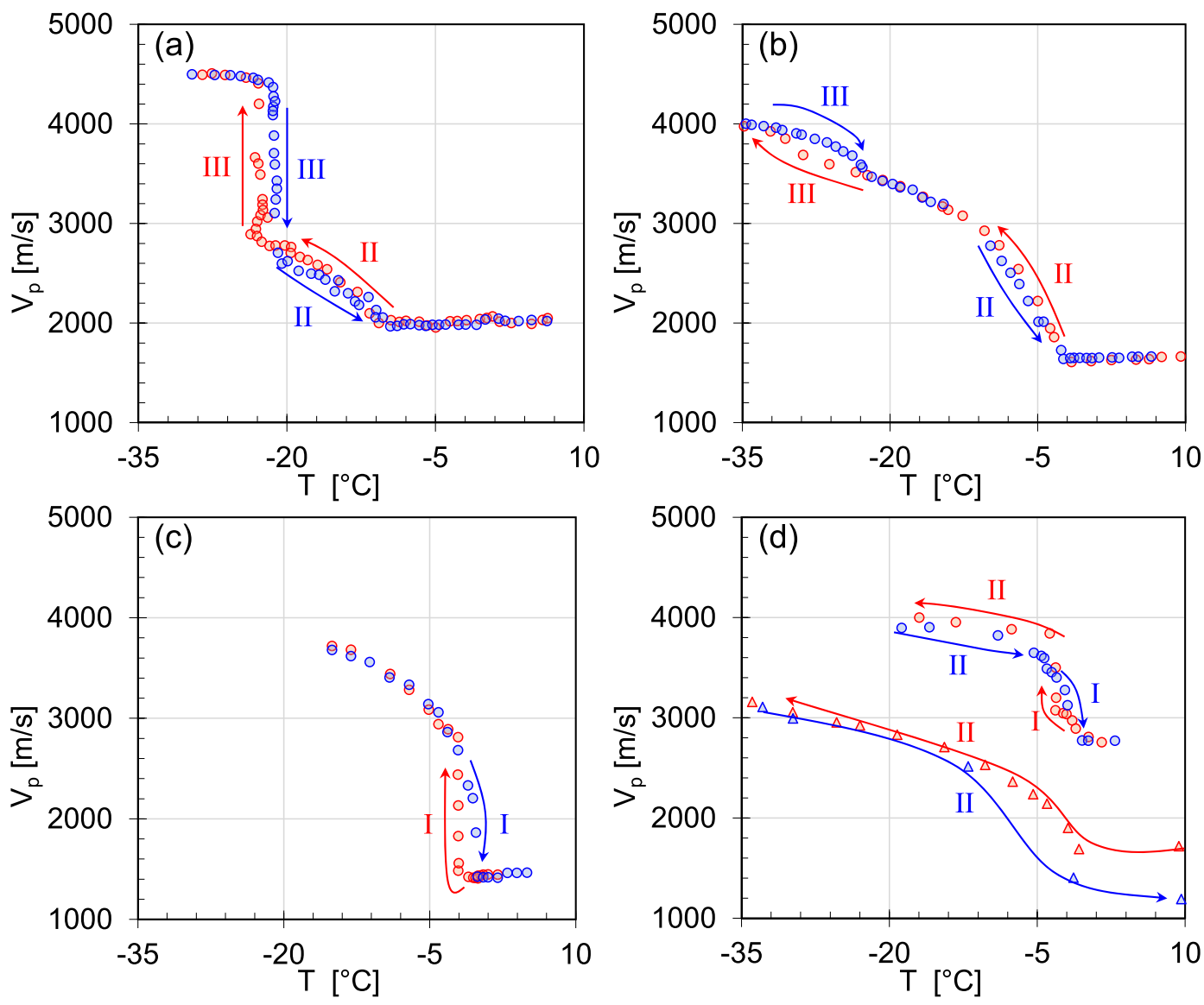

Figure 3. Effect of freezing and thawing on $P$ wave velocity $V_{p}$. Red and blue colors represent freezing and thawing process, respectively. (a) brine-saturated coarse sand $(S=146.3 \mathrm{~g} / \mathrm{L})$ (Dou et al., 2016); (b) silty clay $(S=41 \mathrm{~g} / \mathrm{L}$ ) (Dou et al., 2016); (c) Goodrich clay ( $S$ unknown) (Nakano et al., 1972); (d) O Hanover silt ( $S$ unknown)

(Nakano et al., 1972), $\Delta$ Boom clay ( $S \approx 5$ g/L) (Thimus et al., 1991). I, II, and III correspond to hysteresis Types I, II, and III discussed in section 2.3.

geometry have been proven to influence the curvature of ice-water interfaces and results in different "inkbottle" effects (Tian et al., 2014). Besides, ice lenses can develop with freezing and water molecules move from bound state inside the pores to free bulk state in the ice lenses, which melts at a higher temperature (Bittelli et al., 2003; Kruse \& Darrow, 2017; Thimus et al., 1991). Both freezing-induced ice lens growth and "ink-bottle" effects lead to a higher UWC during freezing than thawing at the same temperature (Kozlowski \& Nartowska, 2013; Tian et al., 2014, 2018; Tice et al., 1989). The acoustic velocity in the freezing process is therefore expected to be lower. However, we observe an opposite trend in all the data sets presented in Figure 3: The acoustic velocity in the process of freezing is higher or similar compared to thawing. We explain this effect by air bubbles that are generated when dissolved air is released from the pore-water during freezing and trapped in the pore ice, if freezing occurs so fast that the air cannot diffuse into the unfrozen water phase as observed by Rowell and Dillon (1972). The process depends on the freezing rate and the amount of air dissolved in the porewater. This accumulated trapped air with freezing may not dissolve (or only partially dissolve) in unfrozen water during thawing, and we hypothesize that this phenomenon cancels or even exceeds the effect of the previous two factors and reduce the $P$ wave velocity.

Type III: electrolyte precipitation- and dissolution-induced hysteresis. Electrolyte precipitation and dissolution may also lead to hysteresis during freezing and thawing near the eutectic temperature of the pore fluid. Carns et al. (2015) found evidence of such precipitation and dissolution hysteresis of hydrohalite $\left(\mathrm{NaCl} \cdot 2 \mathrm{H}_{2} \mathrm{O}\right)$ during formation of sea ice through measurements of translucency changes in brine 
inclusions of thin sections for natural and laboratory grown sea ice during freezing-thawing cycles. They found that dissolution always occurred close to the theoretical eutectic temperature, while precipitation happened at different (lower) temperatures for individual brine inclusions, likely due to the chance formation of single crystals that act as nucleation points for complete precipitation in the inclusion. Carns et al. (2015) suggest that the delay in precipitation is related to brine inclusion size, with smaller inclusions less likely to develop crystallization nuclei and thus more likely to result in supersaturation and delayed precipitation. Here we propose a similar hysteresis process due to supersaturation and delayed precipitation for freezing soils.

Figure 3a illustrates such hysteresis effect for a sand sample where the acoustic velocity abruptly increases (and thus the UWC decreases) at a temperature of approximately $-24^{\circ} \mathrm{C}$, while the reverse process during thawing happens at a higher temperature (roughly $-21^{\circ} \mathrm{C}$ in Figure 3a). Although Dou et al. (2016) explained this effect due to a supercooling type hysteresis (our Type I hysteresis), we suggest that it is more likely due to a supersaturation effect and delayed precipitation during cooling (our Type III hysteresis).

We also observe that this hysteresis occurs more gradually for the fine-grained soil in Figure 3b. Following the arguments of Carns et al. (2015), we propose that the smaller pore sizes of the fine grained soils result in smaller brine inclusions, which would be less likely to develop crystallization nuclei, and thus more likely to result in supersaturation and delayed precipitation.

\section{Review of Geoacoustic Models for UWC Estimates}

This section reviews eight geoacoustic models previously applied for permafrost engineering or in research of similar materials like gas hydrate bearing sediments. We discuss the background and assumptions and the input parameters and especially highlight the physical limitations of each model. The exact formulation (equations) of each model is presented in Appendix A. One of common assumptions for these tested geoacoustic models is that the wavelength is much longer than the characteristic dimensions in the medium such as grains, pores, and ice lens in the frozen soil (continuous medium assumption). The validation of this assumption for the measurement results used in this paper will be discussed in section 4, and the possible dispersion effect induced by different applied wave frequencies will be under consideration in section 5 .

Weighted equation (WE model): The WE model is a weighted average of the three-phase Wood and the time-averaged equations (Timur, 1968; Wyllie et al., 1958). Both models relate the bulk $P$ wave velocity of frozen soils to the velocities of the three individual phases (soil grains, water, and ice) given the density, stiffness of each phase, porosity, and the UWC. The physical meanings and limitations are summarized as follows:

1. In the time-averaged equation, the system slowness (reciprocal of the velocity) is the weighted slowness sum of the constituents in the system. Thus, it is more appropriate for fully cemented (frozen) systems. The Wood's equation, taken as the weighted sum of kinetic energy for constituents, is more proper for particle suspensions (Lee et al., 1996). Here, ice crystal growth is considered analogous to a consolidation process, where unconsolidated sediments change from suspension state to eventually a consolidated state as the UWC decreases (Lee et al., 1996). As discussed by Deschatres et al. (1988), the Wood equation seems to be more accurate at temperatures near $0^{\circ} \mathrm{C}$, while the time-averaged equation is better at low temperatures for unconsolidated permafrost.

2. One empirical factor, $W$ (unitless), determines the preference mode (i.e., cementation or suspension) of the sample in the unfrozen state. Another empirical parameter, $n$ (unitless), controls the "state" shift with freezing and simulates lithification or consolidation effects with crystal growth (Lee et al., 1996). An increase in $n$ results in a faster freezing-induced "consolidation" process from the suspension state. However, both parameters $W$ and $n$ are derived empirically by data fitting, so the values can be applied only to data sets where the sediments are of similar type to those used to determine them. Lee et al. (1996) suggest $n=1$ and $W=1$ for samples of unconsolidated permafrost based on the UWC estimate consistency of $P$ and $S$ wave velocities measured by Zimmerman and King (1986) and King et al. (1988). The porosity of the tested permafrost samples in the two studies was also limited to a range between 0.35 and 0.45 . Besides, factors such as effective pressure and particle aspect ratio cannot be considered in the WE model (Chand et al., 2004; Lee, 2002). 
Zimmerman and King's model (KT model): This model is extended from the two-phase theory developed by Kuster and Toksöz (1974) in order to simulate the elasticity of unconsolidated permafrost (King et al., 1988; Zimmerman \& King, 1986). In case of frozen soils, unconsolidated permafrost is described as a material consisting of spherically shaped quartz/clay grains embedded in a matrix material combining ice frame with spherically shaped water inclusions. The model has been widely applied due to the limited number of parameters needed (Hauck et al., 2011; King et al., 1988; Kneisel et al., 2008). More details regarding the physical limitations are presented here:

1. The KT model is capable of estimating velocity for a narrow range of porosities between $30 \%$ to $50 \%$ and low unfrozen water saturation (UWS) below $60 \%$, because it assumes a dilute concentration of the water inclusions, in other words, a discontinuous water phase in the frozen soils. This assumption may be poor or invalid for frozen soils of high UWC. The stiffness estimate of water-ice mixture based on this assumption approximately corresponds to the upper Hashin-Shtrikman (HS) bound in contrast to spherical ice inclusions in water as lower bound (King et al., 1988). Besides, ice growth increases the bulk modulus through replacing the water phase that can exist inside the pore space and around soil grain surface. It indicates a crystal growth mode in the KT model distinct from grain coating and matrix supporting (Guerin et al., 1999).

2. Zimmerman and King (1986) only considered the spherical shape for water and soil phases of unconsolidated permafrost. The results may therefore strongly deviate from the theoretical effective moduli with high concentration of low aspect ratio inclusion. Fortunately, the geometry influence is minimized for "hard" inclusions like soil particles embedded into "soft" matrix like ice-water mixture.

Pore-scale crystal growth: A group of models referred as pore-scale crystal growth models have been developed to estimate the acoustic velocity of gas hydrates according to the possible crystal growth pattern in the pore space (Dvorkin et al., 1994, 1999; Ecker et al., 1998; Helgerud et al., 1999; Kleinberg \& Dai, 2005). The cementing model assumes that hydrate crystals preferentially grow at grain contacts. Hydrates are described as a uniform coating around the grains in the grain-coating model. In the matrix supporting model, hydrates grow as a new component and come in contact with the soil particles, while the accumulation of hydrates occurs in the pore space without contact with soil grains in the pore-filling model. The models mentioned above facilitate the understanding of crystal growth, which is also relevant for frozen soil. However, compared with the WE and KT models, the crystal growth models require some extra information such as the coordination number, tortuosity, and effective stress. It is also clear that multiple crystal growth patterns could simultaneously take place in freezing process (Pan et al., 2019; Waite et al., 2009).

Two-end member models: In order to cooperate multiple crystallization modes, two-end member models combine different scenarios, for example, a weighted average of the cementing and pore-filling models (Carcione \& Tinivella, 2000; Schindler et al., 2017). However, the two assumed crystal growth modes (cementing and pore filling) lack sound experimental observations, and an empirical weighting factor needs to be calibrated like the WE model. This model combination will not be considered further in this work.

Another mixing strategy combines a completely frozen (ice filled) and a completely unfrozen (water filled) soil model as the stiff and soft end members (Dou et al., 2017; Minshull et al., 1994). In the model proposed by Dou et al. (2017), the stiff end member model implements the self-consistent approximation (SCA) where ice is considered as penny-shaped inclusions in a soil matrix. The soft end member model implements the Herz-Mindlin contact model and Biot theory. Some physical limitations regarding this model are discussed below:

1. The model formulations requires information of the aspect ratio of ice crystals (stiff end member) and the tortuosity of the pore space (soft end member), but the model is not sensitive to these parameters over reasonable parameter ranges (Dou et al., 2017).

2. Ice growth in the model is between the matrix supporting and grain coating modes, which is realistic in most of situations. Thus, the model adopts the HS average as a mixing strategy to combine the two-end elements, while HS average lacks a physical meaning. For example, the HS average fails to model diverse velocity-porosity relation for sandstones (Avseth et al., 2010). Similarly, it seems also not capable of modeling various velocity versus UWS trends for unconsolidated permafrost presented in this paper. 
Biot theory: It was originally developed to describe wave propagation in a fully saturated porous medium (Biot, 1956a, 1956b; Carcione et al., 2010). Leclaire et al. (1994) extended the two-phase Biot theory to a three-phase theory to study frozen porous media and assume no direct contact between solid grains and ice inclusions, by imposing a soil grain-water meniscus-ice crystal sandwich structure. Skeleton strengthening due to the cementing effect of crystal growth has been introduced to improve this three-phase Biot theory (Carcione \& Tinivella, 2000; Gei \& Carcione, 2003). The model considers the influence of effective pressure and energy dissipation mechanisms for wave propagation in the frozen medium. However, it requires a large number of material properties such as soil and hydrate matrix permeability and friction coefficients, which are difficult or even impossible to measure. Furthermore, some empirical factors do not have clear physical meaning and must be determined by data fitting. Therefore, it is difficult to validate this extended three-phase Biot theory. Instead, Lee (2002) modified the Biot-Gassmann theory, here referred to as the BGTL model, by updating the original two-phase Biot theory. It assumes that the $V_{s} / V_{p}$ ratio of a consolidated sediment is related to the $P$ and $S$ wave velocity ratio of the matrix material and the effective porosity of the soil. This assumption is not valid when grains lose their contacts and the sediment $V_{S} / V_{p}$ ratio approaches 0 in a high porosity range. The BGTL model considers gas hydrates and soil grains as one solid composite and calculates the effective solid matrix properties based on the Hill's average equation (Hill, 1952). Lee (2002) proposes an empirical relation between the Biot coefficient and porosity for hydrate sediments, but the porosity of the tested samples falls in a limited porosity range ( 0.3 to 0.4$)$, and data in the low porosity range (less than 0.2 ) are quite scarce. He also suggests that the relationship could be derived based on velocity estimates from the WE model or pore filling model (effective medium theory). Using such model-derived estimates will of course propagate the challenges of using the WE model and pore filling theory.

\section{Comparison Study}

\subsection{Selection of Geoacoustic Models and Data for Comparison Study}

There are only few available papers comparing several geoacoustic models for unconsolidated permafrost (Carcione \& Seriani, 1998; Thimus et al., 1991). Thimus et al. (1991) made a comparison between the three-phase Wood's equation, the time-averaged equation, and KT model for Boom clay. They used NMR and calorimetry techniques to measure relatively accurate UWC values. Carcione and Seriani (1998) tried to verify more models including the three-phase Biot theory proposed by Leclaire et al. (1994). However, the UWC used for verification was estimated by a thermodynamic relationship, which mainly considers capillary effect and assumes Gaussian porosimetric distribution. This relationship might not work for fine-grained permafrost soils. More efforts have been put on geoacoustic model studies and their comparisons for gas hydrate bearing sediments that show similarities with frozen soil (Chand et al., 2004; $\mathrm{Hu}$ et al., 2010; Kim et al., 2013; Konno et al., 2015; Pan et al., 2019; Waite et al., 2009). However, the preparation of synthesized hydrate samples can be quite different such as gas plus unsaturated sediments, gas plus saturated sediments, and gas plus sediments with seed ice (Waite et al., 2009; Yun et al., 2007). Different hydrate nucleation and growth processes in these preparation methods can result in distinct crystal growth habits and nonunique relations between hydrate saturation and acoustic velocity. Thus, conclusions draw from hydrate sediment studies should not be uncritically applied to unconsolidated permafrost. Besides, some measurement methods of hydrate saturation, such as the electrical resistivity method, are indirect and could not provide accurate "reference" results to verify these models (Chand et al., 2004).

This paper first considers the references that contain both acoustic results and UWC measured by NMR or time domain reflectometry (TDR) (Christ \& Park, 2009; Matsushima et al., 2016; Thimus et al., 1991). NMR is considered as one of the most accurate methods for UWC determination (Li, 2009; Tice et al., 1989), since NMR signals, at the resonant frequency of hydrogen nuclei, are proportional to the amount of hydrogen atoms. TDR is an indirect method to measure soil moisture content through the bulk soil dielectric permittivity. It has been proven that TDR can provide UWC estimates with high accuracy after proper calibration and installation (Smith \& Tice, 1988). We also have collected laboratory $V_{p}$ and $V_{s}$ test results and use the $V_{s} / V_{p}$ ratio to validate the consistency of the geoacoustic models. The frequencies applied in the collected acoustic measurements in this paper are listed in Table 1. The highest frequency was $2 \mathrm{MHz}$ with a wavelength from 1 to $3 \mathrm{~mm}$. The wavelength is thus much larger than the coarsest grain size considered 
Table 1

Information of Analyzed Frozen Soil

\begin{tabular}{|c|c|c|c|c|c|c|}
\hline Soil & $n$ & $C_{c}$ & $S(\mathrm{~g} / \mathrm{L})$ & $f(\mathrm{kHz})$ & Freezing method & Reference \\
\hline Sand & 0.29 & 0 & 0 & 2000 & 1-D freezing & Christ and Park (2009) \\
\hline Silt sand & 0.32 & 0.05 & 0 & & & \\
\hline Silt & 0.39 & 0.02 & 0 & & & \\
\hline Unconsolidated sand & 0.41 & 0 & 20 & 1000 & n.d. & Matsushima et al. (2016) \\
\hline Overconsolidated Boom clay & 0.40 & 0.51 & $\approx 5$ & 1000 & Liquified nitrogen freezing & $\begin{array}{l}\text { Thimus et al. (1991); } \\
\text { Nguyen et al. (2013) }\end{array}$ \\
\hline Marine sand (1) (2) & 0.40 & 0.05 & $1.17 / 4.68$ & $500-850$ & Natural permafrost & King et al. (1982) \\
\hline River silt & 0.36 & 0.20 & n.d. & & & \\
\hline River sand & 0.37 & 0.05 & n.d. & & & \\
\hline Marine clay & 0.43 & 0.51 & 17.55 & & & \\
\hline Peking fine sand & 0.32 & n.d. & n.d. & 1000 & Air freezing in the confined container & Wang et al. (2006) \\
\hline Lanzhou loess & 0.34 & n.d. & n.d. & & & \\
\hline Harbin clay & 0.46 & n.d. & n.d. & & & \\
\hline
\end{tabular}

Note. $n$, porosity; $C_{c}$, clay content; $S$, salinity; and $f$, applied frequency.

(fine-grained sand with a maximum grain diameter of $0.1 \mathrm{~mm}$ ). In principle, ice lenses or inclusions at this scale could form during the sample freezing process, but no information is available in the original sources, and we have thus assumed homogeneous samples, where the continuous medium assumptions are valid.

When selecting the geoacoustic models, we used the three following guiding principles: (1) The models should share as many physical parameters as possible. (2) The model parameters were calibrated to fit particular data sets in previous study and contain clear physical meaning. (3) The model has a limited number of input parameters. According to these selection criteria, we finally choose the WE model (Lee et al., 1996), the KT model (Zimmerman \& King, 1986), the pore-scale crystal growth model (Helgerud et al., 1999; Kleinberg \& Dai, 2005), the BGTL model (Lee, 2002), and two-end member model (Dou et al., 2017). Investigated pore-scale crystal growth modes include the grain cementing, grain coating, matrix supporting, and the pore filling. Three-phase Biot theory is out of the scope of this paper since it involves some parameters that are difficult to be determined such as frozen soil permeability.

\subsection{Data Selection and Analysis}

Two sets of measurements are used to make comparison among these selected models: UWC versus acoustic velocity $\left(V_{p}\right.$ and $\left.V_{s}\right)$ in Figure 4 and $V_{s}$ versus $V_{p}$ in Figure 5. This paper only selects the results for which UWC is measured by TDR or NMR, and the soil is well characterized (Christ \& Park, 2009; Matsushima et al., 2016; Thimus et al., 1991). However, there are only five data sets fulfilling this requirement, so joint $V_{p}$ and $V_{s}$ measurement results are also selected to enrich data sets (Christ \& Park, 2009; Domenico, 1984;

Table 2

Material Properties for Frozen Soil

\begin{tabular}{lccccc}
\hline Material & $K(\mathrm{GPa})$ & $G(\mathrm{GPa})$ & $\rho\left(\mathrm{kg} / \mathrm{m}^{3}\right)$ & $V_{p}(\mathrm{~m} / \mathrm{s})$ & $V_{S}(\mathrm{~m} / \mathrm{s})$ \\
\hline Water & $2.25^{\mathrm{a}}$ & 0 & $1,000^{\mathrm{a}}$ & 1,500 & 0 \\
Quartz & $37^{\mathrm{b}}$ & $44^{\mathrm{b}}$ & $2,650^{\mathrm{c}}$ & 6,008 & 4,075 \\
Clay & $20.9^{\mathrm{c}}$ & $6.85^{\mathrm{c}}$ & $2,580^{\mathrm{c}}$ & 3,412 & 1,630 \\
Ice & $8.4^{\mathrm{d}}$ & $3.7^{\mathrm{d}}$ & $920^{\mathrm{d}}$ & 3,807 & 2,005 \\
$C N$ & $\varphi_{\mathrm{c}}$ & $\tau$ & $\alpha$ & $W$ & $n$ \\
$9^{\mathrm{e}}$ & $0.37^{\mathrm{e}}$ & $1^{\mathrm{f}}$ & $0.01^{\mathrm{f}}$ & $1 / 1.5^{\mathrm{d}}$ & $1^{\mathrm{d}}$ \\
\hline
\end{tabular}

Note. $K$, bulk modulus; $G$, bulk modulus; $\rho$, density; $V_{p}, P$ wave velocity; $V_{S}, S$ wave velocity; $C N$, coordination number; $\varphi_{c}$, critical porosity; $\tau$, tortuosity; $\alpha$, aspect ratio; $W$ and $n$, empirical factors.

${ }^{a}$ Leclaire et al. (1994). ${ }^{b}$ Zimmerman and King (1986). ${ }^{\mathrm{c}}$ Chand et al. (2004). ${ }^{\mathrm{d}}$ Lee et al. (1996). ${ }^{\mathrm{e}}$ Helgerud et al. (1999). ${ }^{\mathrm{f}}$ Dou et al. (2016). 

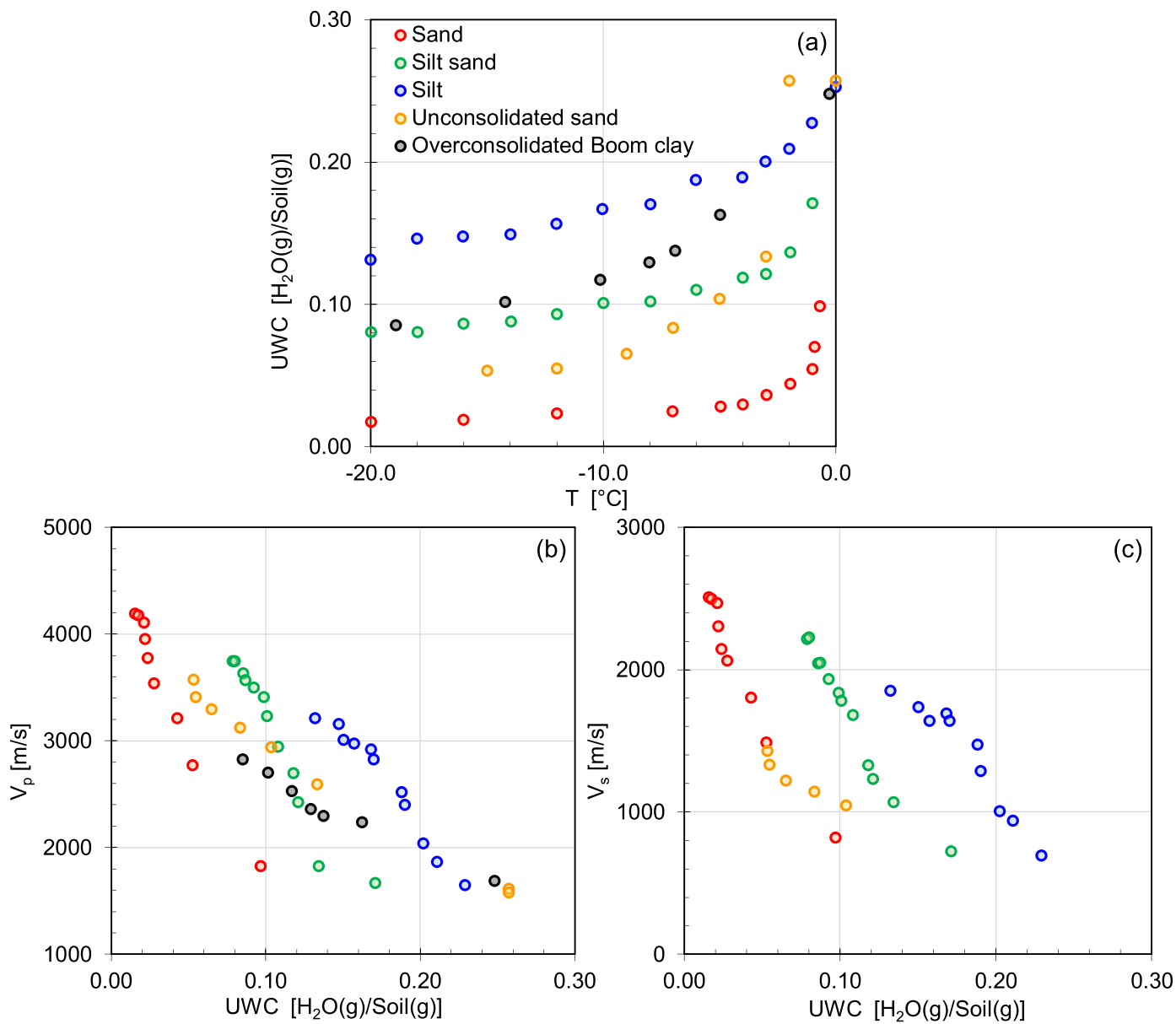

Figure 4. Effect of unfrozen water on acoustic velocity in frozen soil. Sand $(S=0 \mathrm{~g} / \mathrm{L})$, silt sand $(S=0 \mathrm{~g} / \mathrm{L})$, and silt $(S=0 \mathrm{~g} / \mathrm{L})$ (Christ \& Park, 2009); unconsolidated sand ( $S=20 \mathrm{~g} / \mathrm{L})$ (Matsushima et al., 2016); Overconsolidated Boom clay $(S \approx 5 \mathrm{~g} / \mathrm{L})$ (Thimus et al., 1991). Information on these soils can be found in Table 1 . UWC results are independently measured by NMR or TDR.

King et al., 1982; Matsushima et al., 2016; Wang et al., 2006). Table 1 summarizes the characterization for the soils presented in Figures 4 and 5. For part of the analysis, we have chosen to normalize the original UWC data to UWS, defined as the volume ratio of unfrozen water to pores (under an assumption of full saturation in the unfrozen state), ranging from $0 \%$ to $100 \%$.

Figure 4a shows that UWC decreases with decreasing temperature, salinity, and fine content. For example, UWC is only 0.02 for sand without salinity at a temperature of $-5^{\circ} \mathrm{C}$, while it is 0.1 for unconsolidated sand with $20 \mathrm{~g} / \mathrm{L}$ salinity and 0.17 for overconsolidated Boom clay with around $5 \mathrm{~g} / \mathrm{L}$ salinity and $51 \%$ clay content (grain size $<0.002 \mathrm{~mm}$ ). Accordingly, the $P$ and $S$ wave velocities show inverse relationships with UWC in Figures $4 \mathrm{~b}$ and $4 \mathrm{c}$.

Figures $5 \mathrm{a}$ and $5 \mathrm{~b}$ present an approximately linear relationship between $V_{p}$ and $V_{s}$ for frozen sand and silt regardless of temperature, porosity, grain size, and salinity. For example, marine sand (1) and (2) with higher salinities ( $S=1.17$ and $4.68 \mathrm{~g} / \mathrm{L}$ respectively) share the same relationship of $V_{s}$ versus $V_{p}$ with nonsaline sand in Figure 5a. When it comes to clay, the $V_{S} / V_{p}$ ratio becomes clearly smaller and the relation tends to be nonlinear suggested in Figure $5 \mathrm{c}$. The nonlinearity may be caused by the dependence of the ice growth mode on the clay content. These linear trends can facilitate the determination of the frozen soil type when joint analysis of $P$ and $S$ wave velocity such as full-waveform inversion is conducted. 

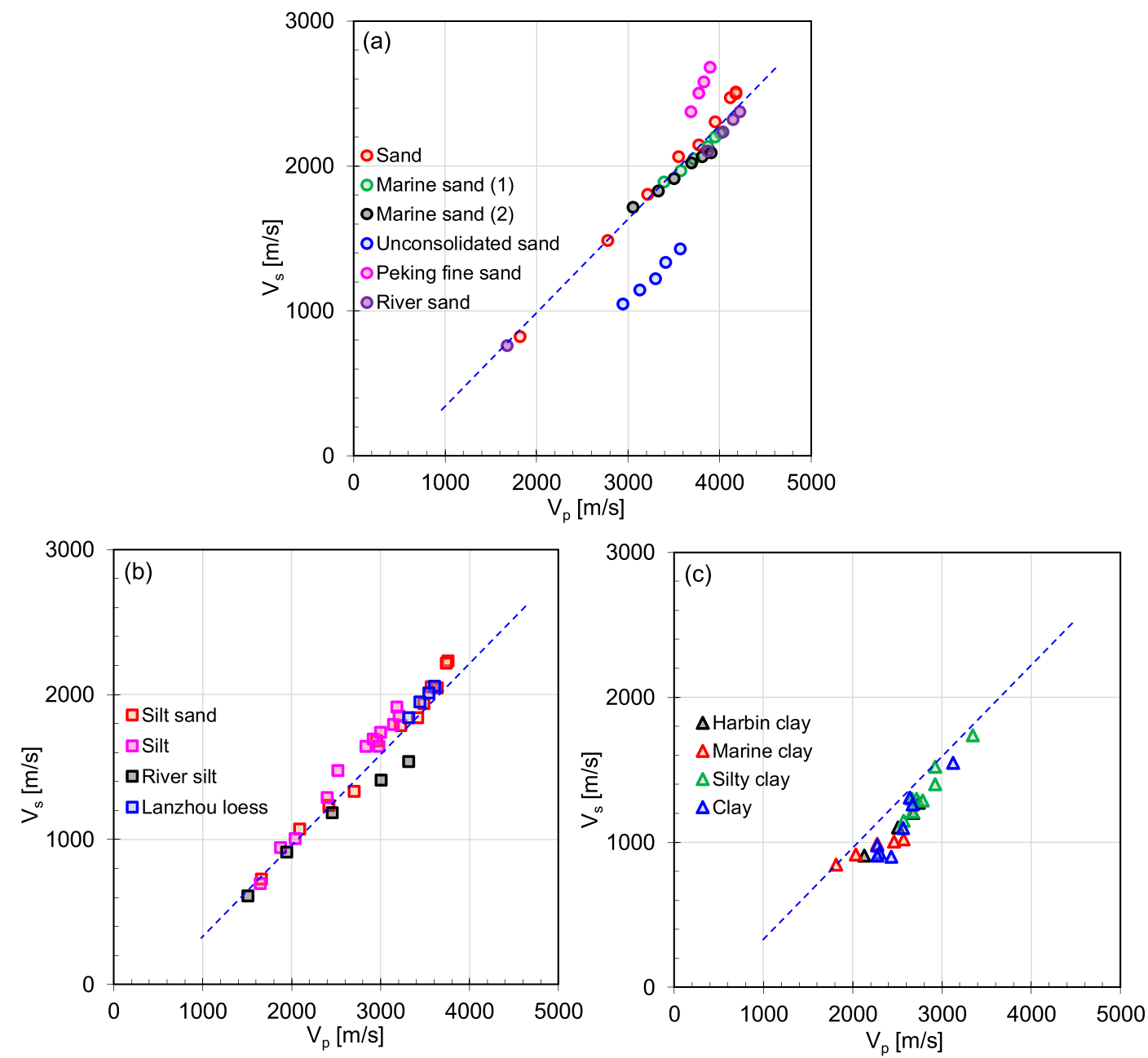

Figure 5. $S$ wave velocity $V_{S}$ versus $P$ wave velocity $V_{p}$ for different types of soil. Sand, silt, sand and silt (Christ \& Park, 2009); unconsolidated sand (Matsushima et al., 2016); marine sand (1), (2), river silt, river sand, and marine clay (King et al., 1982); Peking fine sand, Lanzhou loess, and Harbin clay (Wang et al., 2006); Silty clay and clay (Zimmerman \& King, 1986). Information on these soils can be found in Table 1. Blue dashed line is the linear fitting of $V_{p}$ versus $V_{S}$ for frozen sand.

\subsection{Model Comparison}

UWS estimates from the pore-scale crystal growth models: None of these models alone can estimate the UWS over the full range of $0-100 \%$ for both coarse- and fine-grained frozen soil in Figures 6a1, 6b1, 6c1, 6d1, and $6 \mathrm{e} 1$ and $7 \mathrm{a} 1,7 \mathrm{~b} 1,7 \mathrm{c} 1$, and 7d1. However, these models can provide the insight of ice growth during the freezing. Thermodynamically, crystallization tends to initialize from the unbound water phase in the pore space, which corresponds to pore filling and matrix supporting mode, and progress to surface bound water with further freezing, which matches grain coating and cementing growth mode. As the models indicate, ice growth of sand in Figure 6a1 tends to start in the form of pore filling and/or matrix supporting. When UWS decreases to roughly $50-60 \%$, the grain cementing or coating mode initializes and finally becomes dominated in the freezing process. Silt sand and silt follow a similar ice growth mode, but the mode change takes place at a higher UWS (around 80\% for silt) from matrix supporting/pore filling dominated to grain coating/cementing controlled in Figures $6 \mathrm{c} 1$ and $6 \mathrm{~d} 1$, because silt sand and silt with higher fine content include less unbound water than sand. It indicates that the unbound water content has significant impact on ice crystallization mode. In the meanwhile, the abrupt transition observed for sand, silt and silty sand is not seen for saline sand (20 g/L, Figure 6b1) and clay (5 g/L, Figure 6b1). It indicates that salinity relaxes this mode transition from pore filling/matrix supporting to grain coating/cementing as discussed in section 2.2. 

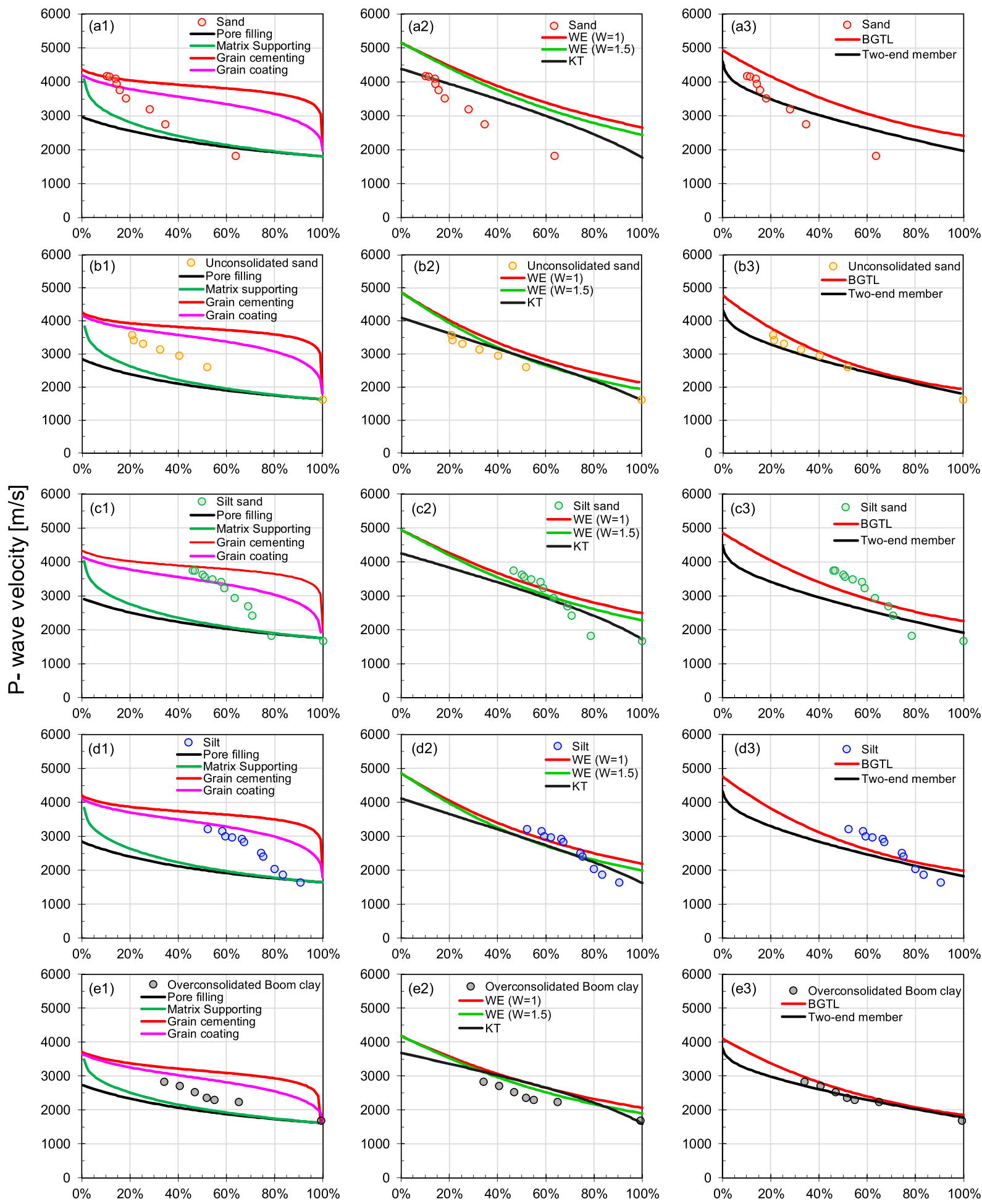

\section{Unfrozen water saturation [\%]}

Figure 6. Comparison of geoacoustic models for $P$ wave velocity of frozen soil according to the measurement of $V_{p}$ versus UWS. Points represent corresponding measurements of $V_{p}$ and UWS (measured by NMR or TDR). Lines represent the $V_{p}$ estimated by various models as a function of UWS based on the parameters given in Tables 1 and 2. Each row corresponds to a different type of soil. For example, (a1)-(a3) correspond to sand, (b1)-(b3) unconsolidated sand, (c1)-(c3) silt sand, (d1)-(d3) silt, and (e1)-(e3) overconsolidated Boom clay. Each column represents a certain set of models. 

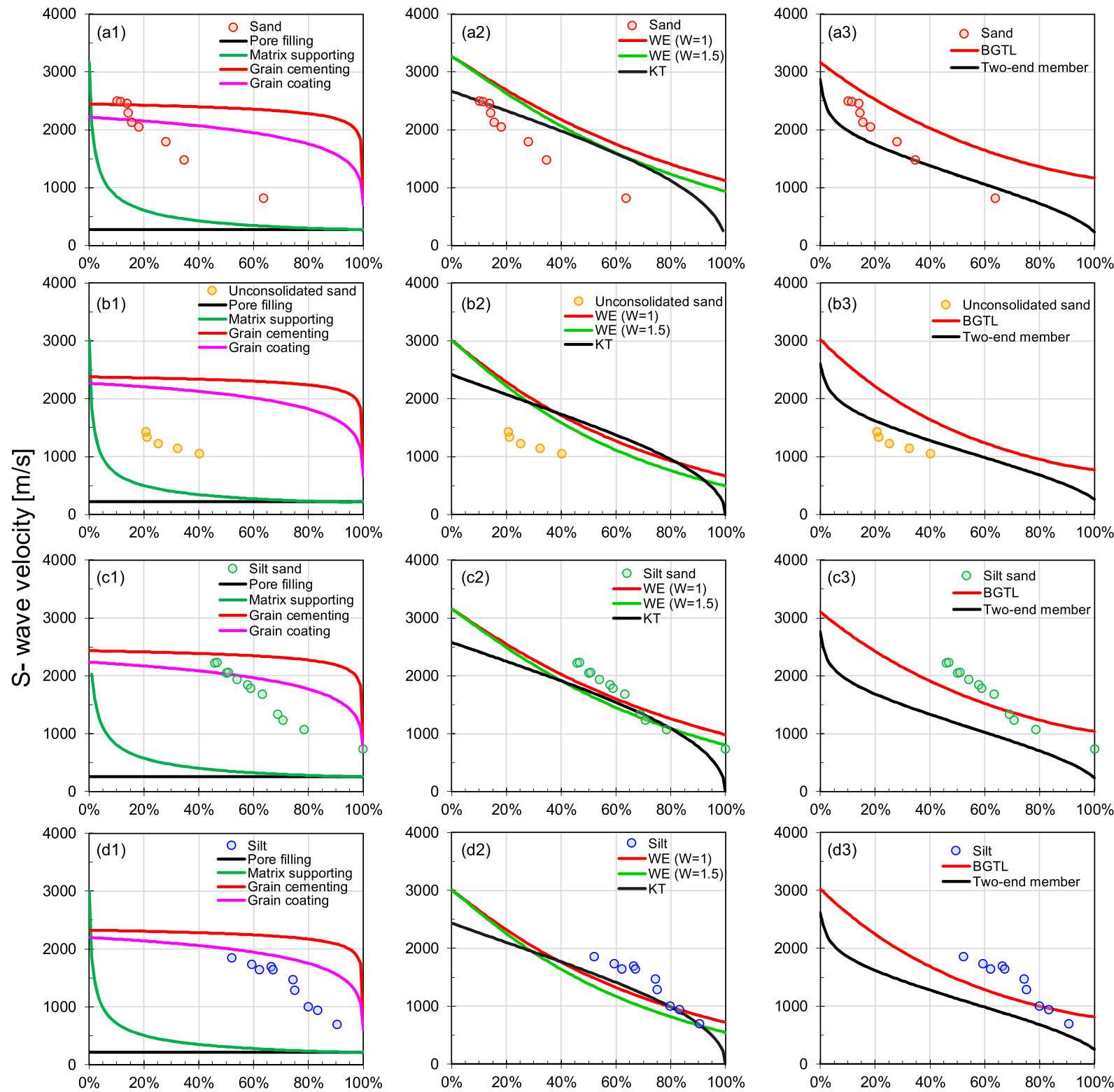

Unfrozen water saturation [\%]

Figure 7. Comparison of geoacoustic models for $S$ wave velocity of frozen soil according to the measurement of $V_{S}$ versus UWS. Points represent corresponding measurements of $V_{S}$ and UWS (measured by NMR or TDR). Lines represent the $V_{S}$ estimated by various models as a function of UWS based on the parameters given in Tables 1 and 2. Each row corresponds to a different type of soil. For example, (a1)-(a3) correspond to sand, (b1)-(b3) unconsolidated sand, (c1)-(c3) silt sand, and (d1)-(d3) silt. Each column represents a certain set of models. The $S$ wave velocity of overconsolidated Boom clay was not reported or measured in Thimus et al. (1991).

Model estimates of UWS from the P wave velocity: The UWS estimates based on the BGTL and two-end member models are quite accurate for unconsolidated sand with 0.41 porosity and overconsolidated Boom clay with 0.4 porosity in Figures $6 \mathrm{~b} 3$ and $6 \mathrm{e} 3$. The average UWS estimate mismatch is only $5 \%$, and the maximum mismatch is $10 \%$. The estimates are less accurate for the WE and KT models. The average mismatch ranges from $8 \%$ to $15 \%$, with a maximum of $20 \%$ overestimation for the WE model in Figures $6 \mathrm{~b} 2$ and 6e2. Both WE and KT models overestimate UWS to some degree. When it comes to silt sand with 0.32 porosity and silt with 0.39 porosity, the WE and KT models can provide slightly better estimates with $10 \%$ average error and a $20 \%$ maximum error in Figures $6 \mathrm{c} 2$ and $6 \mathrm{~d} 2$ compared with the BGTL and two-end member models in 

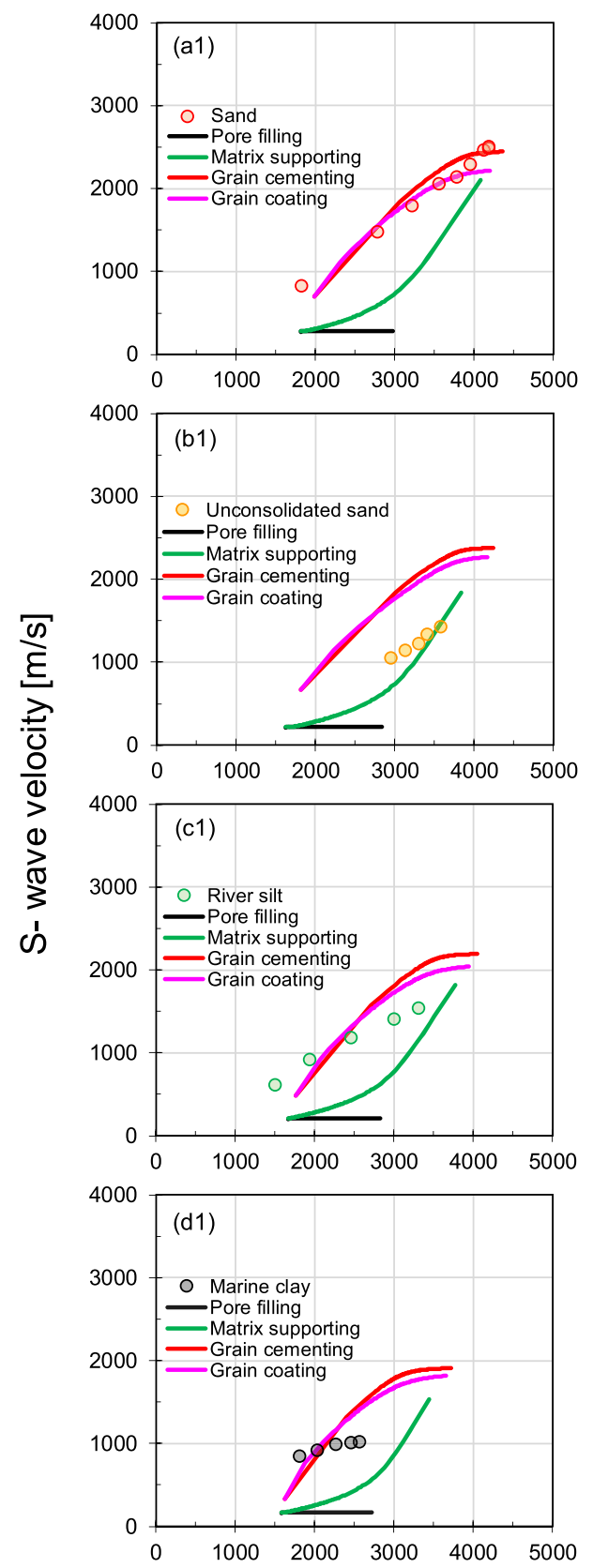

\section{P- wave velocity $[\mathrm{m} / \mathrm{s}]$}

Figure 8. Comparison of geoacoustic models regarding different types of soil according to the measurement of $V_{S}$ versus $V_{p}$. The model curves (lines) are corresponding $V_{p}$ and $V_{s}$ calculated based on variation of UWS from 0 to $100 \%$ (high to low $V_{p}$ or $V_{S}$ ). Points represent consistent measurements of $V_{p}, V_{S}$, and UWS. Each row corresponds to a different type of soil. For example, (a1) and (a2) correspond to sand, (b1) and (b2) unconsolidated sand, (c1) and (c2) river silt, and (d1) and (d2) marine clay. The $V_{S}$ versus result of overconsolidated Boom clay is not presented because of missing $S$ wave velocity measurements in Thimus et al. (1991).

Figures $6 \mathrm{c} 3$ and $6 \mathrm{~d} 3$. There is underestimation at the range from $40 \%$ to $70 \%$ UWS and overestimation from $70 \%$ to $100 \%$ UWS for those models. None of the tested models can well estimate UWS above $20 \%$ for sand with 0.29 porosity in Figures $6 \mathrm{a} 2$ and 6a3. Maximum errors occur at the initialization of ice crystal growth (high UWS) and can be up to $40 \%$ for most of the tested models and even more than $50 \%$ for the WE model. 
Model estimates of UWS from the $S$ wave velocity: The accuracy of UWS estimates based on $V_{S}$ measurements in Figures 7a2, 7a3, 7c2, and 7c3 is comparable to $V_{p}$-based estimates for sand and silt sand. However, the UWS model estimates from $V_{s}$ are significantly worse than those from $V_{p}$ for silt and unconsolidated sand in Figures $7 \mathrm{~b} 2,7 \mathrm{~b} 3,7 \mathrm{~d} 2$, and $7 \mathrm{~d} 3$. The $V_{s}$-based estimate error can be around $20 \%$ for silt and $30 \%$ for unconsolidated sand on average (although relatively few points are available for this sample). Thus, the UWS estimated by these models from $S$ wave velocities is less accurate than predictions based on the $P$ wave velocity, likely because the $S$ wave velocity can be sensitive to other parameters such as effective stress and original cementing among the soil particles other than UWS only. For example, the $V_{S}$ of silt sand in completely unfrozen saturated state is above $700 \mathrm{~m} / \mathrm{s}$ (likely due to cementing), which is much faster than model estimates at the low confinement stress level. However, it is not easy to estimate the effective stress and the cementing based on the information given in the data references and these factors mainly influence the accuracy of the UWS estimates based on $S$ wave velocity.

Model estimates of UWS from $V_{s}$ versus $V_{p}$ : The sand, unconsolidated sand, river silt, and marine clay are selected because our previous analysis (Figure 5) show that sand and silt roughly share a linear relationship of $V_{s}$ versus $V_{p}$ regardless of salinity, temperature and porosity. The $V_{s}$ versus $V_{p}$ estimates based on the KT and BGTL models roughly match the measurements for sand in Figure 8a1, which means that both models provide consistent UWS estimates based on $P$ and $S$ wave acoustic measurements. The WE and two-end member models show a slight mismatch and UWS estimates based on $V_{p}$ measurements are slightly higher than those based on $V_{s}$ measurements. Generally, the KT, BGTL, WE, and two-end member models can provide quite consistent UWS estimates based on $V_{S}$ and $V_{p}$ for all tested sands and silts in Figures 5a and 5b except unconsolidated sand in Figure 8b2. With increasing fine content, the consistency of UWS estimates worsens and the data sets for river silt with $20 \%$ fine content (grain size $<0.075 \mathrm{~mm}$ ) and marine clay with $51 \%$ clay content cannot match the $V_{s}$ versus $V_{p}$ estimate curves anymore. From a combined analysis of Figures $6-8$, it is evident that the fact that a model provides consistent estimates of UWC based on $V_{p}$ and $V_{s}$ measurements is not an indication that this estimate is accurate (compares favorably with measured UWS). This is illustrated in Figure 8 by the grain coating and cementing models, which correlate well with observations of $V_{s}$ versus $V_{p}$ sands and silts, although both models cannot estimate UWS based on $V_{p}$ or $V_{s}$ alone, as shown in Figures 6 and 7. We find that misunderstandings or even incorrect conclusions may arise from validating geoacoustic models based on the joint analysis of $V_{s}$ and $V_{p}$ data sets alone.

\section{Discussions}

In the present study, by comparing data from multiple sources and sample types, we can document the effects of both grain size and salinity on ice crystallization and compare the performance of different models. It is the main difference from most previous studies that only compares one or few models based on few soil types and nonreliable UWC measurement results. In this section, we focus on the physical interpretation of match and mismatch between laboratory results and theoretical models. Besides, possible dispersion effect is also generally discussed.

$S$ wave velocity estimate: The effective stress is one of the key parameters in some of the compared models (the pore filling, matrix supporting, and two-end member models). In this work we have assumed an effective stress of $1 \mathrm{kPa}$ (no load on sample) as the stress level was expected to be low. Although higher values of effective stress can be used to achieve a better $S$ wave velocity match for fully unfrozen soils, there is no evidence to support this adjustment regarding the experimental setups. Besides, none of the crystal growth models alone provide reasonable $S$ wave velocity based estimates over the entire range of UWS, and the HS average applied in the two-end member model has little physical meaning. When it comes to the BGTL model, the $V_{s} / V_{p}$ ratio of sediment is less than the multiplication of $V_{s} / V_{p}$ ratio of the solid phases with the solid fraction for studied unconsolidated permafrost, but they are assumed to be equal in the model. This is the reason that the BGTL-model overestimates $V_{S}$ in most tested conditions. Another error source is from the Biot coefficient estimate. The empirical Biot coefficient versus porosity relation in the BGTL model proposed by Lee (2002) is higher than the estimate they derive from the grain coating or cementing models at a given UWS. When the ice growth mode quickly transforms from matrix supporting and pore filling to grain coating/cementing, the $S$ wave velocity estimated based on the BGTL model is less than actual 

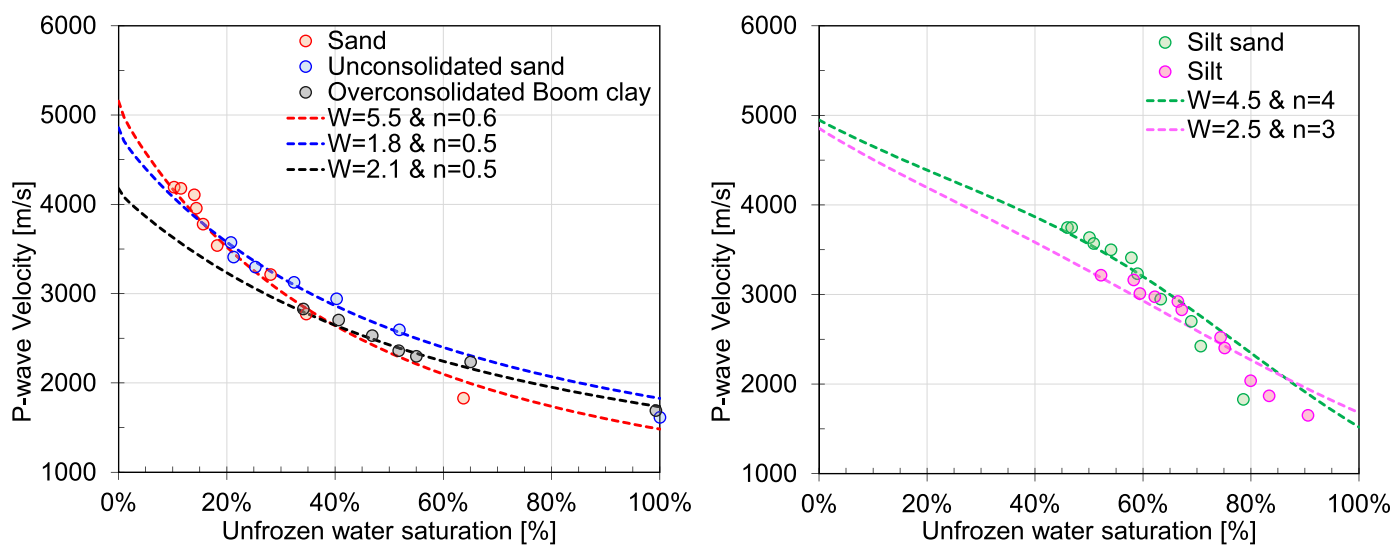

Figure 9. $W$ and $n$ values for data fitting of $P$ wave velocity versus UWS. Points represent measured data of $V_{p}$ and UWS (measured by NMR or TDR), lines represent model predictions of $V_{p}$ based on variation of UWS with the following choices for $W$ and $n$ : sand: $W=5.5$ and $n=0.6$ (red); unconsolidated sand: $W=1.8$ and $n=0.5$ (blue); overconsolidated Boom clay: $W=2.1$ and $n=0.5$ (black); silt sand: $W=4.5$ and $n=4$ (green); sand: $W=2.5$ and $n=3$ (pink).

measurements for, for example, silt and silt sand (see Figures 7c3 and 7d3). $S$ wave velocities derived by the WE model completely depends on the empirical $V_{p}-V_{s}$ relation developed for mudrocks by Castagna et al. (1985). New $V_{p}-V_{S}$ relations for unconsolidated permafrost might be concluded from Figure 5.

$P$ wave velocity estimate: The pore filling and matrix supporting models perform relatively good for sand, silt, sand and silt at the initialization of ice crystal growth (high UWS), and the grain coating/cementing models give reasonable values for the high ice content limit (low UWS). However, single crystal growth mode throughout the whole freezing process has not been found for any tested frozen soils, which is different from the crystal growth behavior in some synthetic hydrate-bearing sediments.

Lee et al. (1996) selected $n=1$ and $W=1$ to fit the joint $P$ and $S$ wave velocities reported by Zimmerman and King (1986) using the WE model. However, as we previously concluded, joint analysis of $P$ and $S$ wave velocities may lead to incorrect conclusions with respect to UWS, and their choice of $n$ and $W$ values was not specifically determined for different soil types and salinities. In order to provide valid choices of $n$ and $W$ specifically for the unconsolidated permafrost, we tested in this paper, Figure 9 shows the good fitting based on the various $n$ and $W$ values. The $n$ value is related to the ice crystal growth modes, and it seems from our results that $n<1$ is suitable for saline soils or soils with little bound water (e.g., coarse grained soils) while $n>1$ for soils with large amount of bound water (e.g., silt sand).

The KT model generally performs better with increasing ice content (lower UWS) since it assumes a dilute concentration of water inclusions. However, the water phase might be continuous at the initialization of ice growth (high UWS), and the KT model may therefore overestimate the UWS when it is above 70\% in most tested soils. However, the KT model can also underestimate UWS when the crystal growth mode quickly shifts to grain coating or cementing, as seen for the silt sand and silt samples at a range of UWS between 40-60\%. The reason is that the KT model essentially suggests the crystallization different from grain coating as we explained in section 3. There is a high consistency between the KT model estimate and measurement results for unconsolidated sand because the sample porosity (0.41) and measured UWS from $20 \%$ to $50 \%$ are located in the well-estimated range of the model (Zimmerman \& King, 1986). The good match is also achieved for overconsolidated Boom clay with more than 50\% clay content although the clay particles are far from spherical shape as assumed by the model. It indicates that the shape effect of "hard" inclusions is not significant.

When it comes to the BGTL model, the empirically defined Biot coefficient versus porosity relationship by Lee (2002) could be the main source of estimate errors. It falls between the value derived from pore filling and matrix supporting as upper bound and the value at the basis of grain coating and cementing as lower bound. Essentially, this proposed relation in the BGTL model estimates the variation of the Biot coefficient with a "smooth" freezing process. This is the reason the BGTL model can achieve a good match for unconsolidated saline sand and overconsolidated Boom clay with gradual freezing process. It can also explain why 
this model overestimates the UWS when pore filling and matrix supporting are the dominating crystal growth modes and underestimates the UWS when crystallization mode quickly shifts to grain cementing and coating like for silt sand and silt.

In the two-end member model proposed by Dou et al. (2017), the upper and lower HS bounds approximately correspond to the grain coating and matrix supporting crystal growth modes. The HS average, as a strategy to mix the fully "unfrozen" and "frozen" ends, can also model a "smooth" $P$ wave velocity versus UWS relationship at the range of $0 \%$ to $100 \%$ UWS similar with BGTL model. That is why it can match the experimental results for unconsolidated sand and overconsolidated Boom clay, since the ions and clay particles relax the freezing process and ice grows more homogeneously, in contrast to the ice growth in frozen nonsaline sand, silt sand, and silt where an abrupt change in crystal growth mode is observed.

Dispersion effect: In order to consider the effect of frequency on wave velocity and further UWS estimate, this paper considers Biot-type velocity dispersion as a result of the decoupling between solid phases (soil particles and ice) and fluid phase (unfrozen water) in the three-phase Biot theory proposed by Leclaire et al. (1994). The characteristic frequency that distinguishes the low- and high-frequency ranges increases from 75 to $300 \mathrm{kHz}$ as UWS decreases from $3.3 \%$ to $1.7 \%$. It indicates that the lowest applied frequency of $500 \mathrm{kHz}$ for the experiments referenced in this paper is in the high-frequency range even for completely frozen soils. Besides, the ratio of high-frequency fast wave velocity to low-frequency velocity approaches 1 as the characteristic frequency increases with freezing. To consider both effects, dispersion of the fast wave can be practically negligible when the wave frequency is larger than $30 \mathrm{kHz}$ (Lee \& Waite, 2008). To sum up, all measured wave velocities in this paper are in the high-frequency domain and dispersion effects can be neglected for UWS estimates.

\section{Conclusions}

This study comprised a review and reanalysis of previously published acoustic velocity measurements for frozen soil and continued with an intercomparison of selected geoacoustic models for UWC or UWS estimate accuracy. It finally ends up with physical interpretations in the discussion. Apart from reviewing previous works, we also draw several original conclusions below:

- Effect of air bubbles formed and trapped during freezing can counteract the hysteresis in acoustic velocities induced by the "ink-bottle" effect and ice lens growth as suggested by acoustic velocity measurement. Furthermore, this study documents a freezing-thawing hysteresis near the eutectic temperature of frozen soil and attribute this to the effect of salt precipitation and dissolution.

- Regardless of porosity, grain size, temperature, and salinity, frozen sand and silt generally share the same linear trend between measured $V_{s}$ and $V_{p}$. Measurement results for frozen clay generally plot below the trend line.

- The joint analysis of classical crystal growth models and UWS versus $V_{p}$ measurements indicates that the growth of ice crystals starts from the unbound water phase in the pore space (pore filling and matrix supporting mode) and grow toward the grain surface with further freezing (grain coating and cementing mode). The UWS at which a shift in ice crystallization mode is observed depends on the unbound water content in the soil. The salinity can smoothen this transition from ice filling/matrix supporting to grain coating/cementing crystallization mode.

- $V_{p}$ is a better measure for estimating UWS in comparison with $V_{s}$. The value of the parameter $n$ highly determines the prediction capacity of the WE model, and it is dependent on the ice crystal growth mode. The KT, BGTL, and two-end member models overestimate the UWS when ice growth is mainly pore filling or/and matrix supporting dominated and water is the continuous phase other than ice, while underestimation can be expected when grain coating/cementing gradually becomes dominating. Satisfactory estimates are made for unconsolidated saline sand and overconsolidated Boom clay mainly because both show quite "smooth" ice crystal growth.

- Most of tested models (grain coating and cementing, KT, BGTL, WE, and two-end member) can also provide quite consistent UWS estimates based on $V_{s}$ versus $V_{p}$ for tested sands and silts. An increase in clay content would deviate the predicted $V_{s}$ versus $V_{p}$ curve from measurement results. However, the fact that a model provides consistent estimates of UWC based on $V_{p}$ and $V_{s}$ measurements does not ensure that the UWC estimate is accurate. Independent measurements of UWC are necessary for model validation. 


\section{Appendix A: Key Equations in the Compared Models}

The key equations of the geoacoustic models reviewed in the main paper are presented in this appendix to provide the full theoretical formulation of the models. The estimated $V_{p}(\mathrm{~m} / \mathrm{s})$ and $V_{s}(\mathrm{~m} / \mathrm{s})$ in the following models (except the WE model) are calculated as follows (Helgerud et al., 1999):

$$
\begin{gathered}
\rho_{m}=C_{c}(1-\varphi) \rho_{c}+\left(1-C_{c}\right)(1-\varphi) \rho_{s}+S_{w} \varphi \rho_{w}+\left(1-S_{w}\right) \varphi \rho_{i} \\
V_{p}=\sqrt{\frac{K+4 / 3 G}{\rho_{m}}} \\
V_{s}=\sqrt{\frac{G}{\rho_{m}}}
\end{gathered}
$$

where $C_{c}$ and $\left(1-C_{c}\right)$ are the clay and quartz fractions (unitless); $S_{w}$ and $\left(1-\mathrm{S}_{\mathrm{w}}\right)$ are the unfrozen water and ice saturations (unitless); $\varphi$ is the soil porosity (unitless); $\rho_{m}, \rho_{c}, \rho_{s}, \rho_{w}$, and $\rho_{i}$ are the mixture, clay, sand, water, and ice densities $\left(\mathrm{kg} / \mathrm{m}^{3}\right) ; K$ and $G$ are the estimated effective bulk and shear moduli of the (partly) frozen soil $(\mathrm{Pa})$.

The HS average suggested by Hill (1952) is used by some models to calculate the effective bulk $K_{S}(\mathrm{~Pa})$ and shear $G_{s}(\mathrm{~Pa})$ modulus of solid phases composed of quartz, clay, and ice (if present).

$$
K_{s}=\frac{1}{2}\left[\sum_{i=1}^{m} f_{i} K_{i}+\left(\sum_{i=1}^{m} f_{i} / K_{i}\right)^{-1}\right], G_{s}=\frac{1}{2}\left[\sum_{i=1}^{m} f_{i} G_{i}+\left(\sum_{i=1}^{m} f_{i} / G_{i}\right)^{-1}\right]
$$

where $m$ is the number of solid components; $f_{i}$ (unitless), $K_{i}(\mathrm{~Pa})$ and $G_{i}(\mathrm{~Pa})$ are the volumetric fraction, bulk, and shear moduli of the $i$ th component in the solid phase, and $m$ is the total number of phases considered.

WE model. The three-phase Wood equation shown in Equation A5 estimates the bulk $P$ wave velocity, $V_{p 1}(\mathrm{~m} / \mathrm{s})$, from the weighted average of the reciprocal of the bulk moduli for water, ice and soil (solid) phases:

$$
\frac{1}{\rho V_{p 1}^{2}}=\frac{\varphi S_{w}}{\rho_{w} V_{w}^{2}}+\frac{\varphi\left(1-S_{w}\right)}{\rho_{i} V_{i}^{2}}+\frac{(1-\varphi)}{\rho_{m} V_{m}^{2}}
$$

where $V_{w}, V_{i}$, and $V_{m}(\mathrm{~m} / \mathrm{s})$ are the $P$ wave velocities of water, ice, and mineral phases, respectively, $\varphi$ is the soil porosity (unitless), $S_{w}$ (unitless) is UWS, $\rho_{w}, \rho_{i}$, and $\rho_{m}\left(\mathrm{~kg} / \mathrm{m}^{3}\right)$ are the densities of water, ice, and mineral phases.

In the three-phase time-averaged equation, $V_{p 2}(\mathrm{~m} / \mathrm{s})$ is derived from the weighted slowness average for water, ice and soil (solid) phases:

$$
\frac{1}{V_{p 2}}=\frac{\varphi S_{w}}{V_{w}}+\frac{\varphi\left(1-S_{w}\right)}{V_{i}}+\frac{(1-\varphi)}{V_{m}}
$$

The $V_{p}$ estimate of the WE model is then the weighted average of $V_{p 1}$ and $V_{p 2}$ by unitless empirical factors $n$ and $W$ :

$$
\frac{1}{V_{p}}=\frac{W \varphi S_{w}{ }^{n}}{V_{p 1}}+\frac{1-W \varphi S_{w}{ }^{n}}{V_{p 2}}
$$

Lee et al. (1996) adopted the empirical relation $V_{s}$ versus $V_{p}$ presented in Equation A8 for mudrock proposed by Castagna et al. (1985) to calculate the $S$ wave velocity $V_{S}$ of frozen soil based on the estimated $V_{p}$ from Equation A7.

$$
V_{s}=0.8621 V_{p}-1172.4
$$


$K T$ model. The KT model estimates the effective bulk $K$ and shear modulus $G$ for a two-phase medium consisting of a continuous matrix (with moduli $K_{m}$ and $G_{m}[\mathrm{~Pa}]$ ) with spherical inclusions (of moduli $K_{i}$ and $\left.G_{i}[\mathrm{~Pa}]\right)$ as follows (King, 1984):

$$
\begin{gathered}
\frac{K}{K_{m}}=\frac{1+\left[4 G_{m}\left(K_{i}-K_{m}\right) /\left(3 K_{i}+4 G_{m}\right) K_{m}\right] c}{1-\left[3\left(K_{i}-K_{m}\right) /\left(3 K_{i}+4 G_{m}\right)\right] c} \\
\frac{G}{G_{m}}=\frac{6 G_{i}\left(K_{m}+2 G_{m}\right)+\left(9 K_{m}+8 G_{m}\right)\left[(1-c) G_{m}+c G_{i}\right]}{G_{m}\left(9 K_{m}+8 G_{m}\right)+6\left(K_{m}+2 G_{m}\right)\left[(1-c) G_{i}+c G_{m}\right]}
\end{gathered}
$$

where $c$ (unitless) is the volumetric fraction of inclusion concentration.

Zimmerman and King (1986) and King (1984) assume the unconsolidated permafrost as spherical soil particles embedded into a matrix composed of spherical water inclusions in a continuous ice phase. This is effectively a three-phase material, and Equations A9 and A10 are applied iteratively to first establish the moduli of the ice-water mixture and then to calculate the moduli of the material consisting of soil grains embedded in the ice-water mixture (while considered a homogenous medium).

Four pore-scale crystal growth models. The matrix supporting, pore filling, grain coating, and cementing models are four phenomenological pore-scale ice crystal growth models developed by (Dvorkin \& Nur, 1996; Helgerud et al., 1999).

The matrix supporting and the pore filling models also adopt Hertz-Mindlin contact theory to calculate dry frame bulk and shear modulus ( $K_{d r y}$ and $\left.G_{d r y}[\mathrm{~Pa}]\right)$ with the consideration of a critical porosity $\varphi_{c}$ (unitless) that distinguishes the consolidation and suspension state of solid particles:

$$
\begin{gathered}
K_{D r y}= \begin{cases}{\left[\frac{\varphi / \varphi_{c}}{K_{H M}+4 /{ }_{3} G_{H M}}+\frac{1-\varphi / \varphi_{c}}{K+4 /{ }_{3} G_{H M}}\right]^{-1}-\frac{4}{3} G_{H M}} & \varphi<\varphi_{c} \\
{\left[\frac{(1-\varphi) /\left(1-\varphi_{c}\right)}{K_{H M}+4 / 3{ }_{3} G_{H M}}+\frac{\left(\varphi-\varphi_{c}\right) /\left(1-\varphi_{c}\right)}{4 / 3}\right]^{-1}-\frac{4}{3} G_{H M}} & \varphi \geq \varphi_{c}\end{cases} \\
G_{D r y}= \begin{cases}{\left[\frac{\varphi / \varphi_{c}}{G_{H M}+Z}+\frac{1-\varphi / \varphi_{c}}{G+Z}\right]^{-1}-Z \varphi} \\
{\left[\frac{(1-\varphi) /\left(1-\varphi_{c}\right)}{G_{H M}+Z}+\frac{\left(\varphi-\varphi_{c}\right) /\left(1-\varphi_{c}\right)}{Z}\right]^{-1}-Z \varphi} & \geq \varphi_{c}\end{cases} \\
Z=\frac{G_{H M}}{6}\left(\frac{9 K_{H M}+8 G_{H M}}{K_{H M}+2 G_{H M}}\right)
\end{gathered}
$$

where $K_{H M}$ and $G_{H M}$ can be calculated by the Equations A14 and A15:

$$
\begin{gathered}
K_{H M}=\left[\frac{C N^{2}\left(1-\varphi_{c}\right)^{2} G_{s}^{2}}{18 \pi^{2}\left(1-v_{s}\right)^{2}} P\right]^{\frac{1}{3}} \\
G_{H M}=\frac{5-4 v_{s}}{5\left(2-v_{s}\right)}\left[\frac{3 C N^{2}\left(1-\varphi_{c}\right)^{2} G_{s}^{2}}{2 \pi^{2}\left(1-v_{s}\right)^{2}} P\right]^{\frac{1}{3}}
\end{gathered}
$$

Here, $P(\mathrm{~Pa})$ is the effective stress, $\mathrm{CN}$ (unitless) is the coordinator number, and $v_{S}$ (unitless) is the solid Poisson ratio. In this study, $P$ is assumed equal to $1 \mathrm{kPa}$, given the nonconfinement testing conditions and self-weight of all the tested samples presented in this paper.

The pore filling crystal growth model assumes the ice grows in a fluid phase as a new component. The bulk modulus of the fluid phase, $K_{f}$, is a weighted reciprocal average of the bulk water $K_{w}$ and ice moduli $K_{i}$ through UWS $S_{w}$ :

$$
K_{f}=\left[\left(1-S_{w}\right) / K_{i}+S_{w} / K_{w}\right]^{-1}
$$

The effective bulk and shear modulus, $K$ and $G$, in the pore filling model are then calculated using Gassmann's equations (Helgerud et al., 1999): 


$$
\begin{gathered}
K=K_{s} \frac{\varphi K_{D r y}-(1+\varphi) K_{f} K_{D r y} / K_{s}+K_{f}}{(1-\varphi) K_{f}+\varphi K_{s}-K_{f} K_{D r y} / K_{s}} \\
G=G_{D r y}
\end{gathered}
$$

The matrix supporting model assumes the ice grows as a solid phase while the fluid phase is only composed of water. It reduces the original porosity $\varphi$ to "effective" porosity $\varphi_{1}=\varphi S_{w}$. The fractions of soil and ice $\left(f_{s}\right.$ and $f_{i}$ [unitless] $)$ in the solid phase require recalculations and can be further used in the Equation A4 to calculate new solid bulk $K_{s}$ and shear $G_{S}$ moduli:

$$
f_{s}=(1-\varphi) /\left(1-\varphi_{1}\right) ; f_{i}=\varphi\left(1-S_{w}\right) /\left(1-\varphi_{1}\right) ;
$$

The Gassmann's Equation A17 and A18 are also used to calculate effective bulk and shear moduli, $K$ and $G$, in the matrix supporting model.

Both the grain coating and the cementing models use the following equations to calculate the effective drained rock moduli:

$$
\begin{gathered}
K_{D r y}=\frac{C N(1-\varphi)}{6} M_{i} S_{n}(\alpha) \\
G_{D r y}=\frac{3 K_{D r y}}{5}+\frac{3 C N(1-\varphi)}{20} G_{i} S_{\tau}(\alpha)
\end{gathered}
$$

where the value of $\alpha$ is different for the cementing model (Equation A22) and the grain coating model (Equation A23) and the $S_{n}$ and $S_{\tau}$ are functions of $\alpha$ and explained by Kleinberg and Dai (2005):

$$
\begin{gathered}
\alpha=2\left[\frac{\left(1-S_{w}\right) \varphi}{3 C N(1-\varphi)}\right]^{0.25} \\
\alpha=\left[\frac{2\left(1-S_{w}\right) \varphi}{3 C N(1-\varphi)}\right]^{0.5}
\end{gathered}
$$

The Gassmann's Equations A17 and A18) are applied again to calculate effective bulk and shear moduli of the grain coating and the cementing models.

Two-end member models. The SCA is suggested by Dou et al. (2017) to calculate the bulk modulus $K_{I F}(\mathrm{~Pa})$ and shear modulus $G_{I F}(\mathrm{~Pa})$ for the fully frozen end member only composed of ice and soils:

$$
\begin{aligned}
& K_{1}+K_{2}=0 \\
& G_{1}+G_{2}=0
\end{aligned}
$$

where $K_{1}$ and $G_{1}$ are the moduli when ice is assumed as penny-shaped inclusions embedded into the continuous soil phase. $K_{2}$ and $G_{2}$ are the moduli when soil grains are assumed to be spherical inclusions built into the ice matrix. Equations A26-A29 can be introduced into Equations A24 and A25, which are solved iteratively for the moduli $K_{I F}$ and $G_{I F}$.

$$
\begin{gathered}
K_{1}=\varphi\left(K_{i}-K_{I F}\right) P_{*_{i}}^{\text {penny }- \text { inclusion }} \\
G_{1}=\varphi\left(G_{i}-G_{I F}\right) Q_{*_{i}}^{\text {penny }- \text { inclusion }} \\
K_{2}=(1-\varphi)\left(K_{s}-K_{I F}\right) P_{*_{s}}^{\text {sphere }} \\
G_{2}=(1-\varphi)\left(G_{S}-G_{I F}\right) Q_{*_{s}}^{\text {sphere }}
\end{gathered}
$$

where $P$ and $Q$ (unitless) are shape factors explained by Dou et al. (2017). 
The bulk modulus $K_{W F}(\mathrm{~Pa})$ and shear modulus $G_{W F}(\mathrm{~Pa})$ of the fully unfrozen end member adopt the Herz-Mindlin contact model and Biot theory (Dou et al., 2017). In the first step, the dry granular frame modulus $K_{g f}$ and $G_{g f}(\mathrm{~Pa})$ are calculated as follows:

$$
\begin{gathered}
v_{s}=\frac{3 K_{s}-2 G_{s}}{2\left(3 K_{s}+G_{s}\right)} \\
K_{g f}=\left(\frac{C N^{2}(1-\varphi)^{2} G_{s}^{2}}{18 \pi^{2}\left(1-v_{s}\right)^{2}} P\right)^{1 / 3} \\
G_{g f}=\left(\frac{5-4 v_{s}}{5\left(2-v_{s}\right)}\right)\left(\frac{3 C N^{2}(1-\varphi)^{2} G_{s}^{2}}{2 \pi^{2}\left(1-v_{s}\right)^{2}} P\right)^{1 / 3}
\end{gathered}
$$

where $v_{S}$ is the Poisson's ratio of the solid grains (unitless).

Biot's fluid substitution is then used to calculate saturated bulk modulus, and detailed formulas for calculation of the factors $\Delta, S, R, Q, \rho_{11}, \rho_{12}, \rho_{22}$, and $\rho_{W F}$, in Equations A33 and A34 can be found in Dou et al. (2017).

$$
\begin{gathered}
V_{P \infty}=\left(\frac{\Delta+\left(\Delta^{2}-4\left(\rho_{11} \rho_{22}-\rho_{12}^{2}\right)\left(S R-Q^{2}\right)\right)^{1 / 2}}{2\left(\rho_{11} \rho_{22}-\rho_{12}^{2}\right)}\right)^{1 / 2} \\
V_{S \infty}=\left(\frac{G_{g f}}{\rho_{W F}-\varphi \rho_{w} \tau^{-1}}\right)^{1 / 2}
\end{gathered}
$$

The bulk modulus $K_{W F}$ and shear modulus $\mathrm{G}_{\mathrm{WF}}$ can be calculated as follows:

$$
\begin{gathered}
K_{W F}=\left(V_{P \infty}^{2}-\frac{4}{3} V_{S \infty}^{2}\right) \rho_{W F} \\
G_{W F}=V_{S \infty}^{2} \rho_{W F}
\end{gathered}
$$

The effective modulus $K$ and $G$ of partially frozen soil can then be estimated as a HS average to mix modified upper ( $K_{H S+}$ and $\left.G_{H S+}\right)$ and lower $\left(K_{H S-}\right.$ and $\left.G_{H S-}\right)$ HS bounds:

$$
\begin{gathered}
K=\frac{1}{2}\left(K_{H S}+K_{H S-}\right) \\
G=\frac{1}{2}\left(G_{H S}+G_{H S-}\right) \\
K_{H S+}=K_{I F}+\frac{S_{w}}{\left(K_{W F}-K_{I F}\right)^{-1}+\left(1-S_{w}\right)\left(K_{I F}+4 / 3 G_{I F}\right)^{-1}} \\
G_{H S+}=G_{I F}+\frac{S_{w}}{\left(G_{W F}-G_{I F}\right)^{-1}+2\left(1-S_{w}\right)\left(\frac{K_{I F}+2 G_{I F}}{5 G_{I F}\left(K_{I F}+4 / 3 G_{I F}\right)}\right)} \\
K_{H S-}=K_{W F}+\frac{1-S_{w}}{\left(K_{I F}-K_{W F}\right)^{-1}+S_{w}\left(K_{W F}+4 / 3 G_{W F}\right)^{-1}} \\
G_{H S-}=G_{W F}+\frac{1-S_{w}}{\left(G_{I F}-G_{W F}\right)^{-1}+2 S_{w}\left(\frac{K_{W F}+2 G_{W F}}{5 G_{W F}\left(K_{W F}+4 / 3 G_{W F}\right)}\right)}
\end{gathered}
$$

BGTL model. In the modified Biot-Gassmann theory proposed by Lee (2002), the Biot coefficient, $\beta$ (unitless), can be empirically obtained as a function of "effective" porosity $\varphi_{1}$ : 


$$
\beta=\frac{-184.0468}{1+e^{\left(\varphi_{1}+0.56468\right) / 0.10817}}+0.99494
$$

A modulus, $M(\mathrm{~Pa})$ describes the pressure needed to change fluid volume without changing formation volume based on Gassmann's theory:

$$
\frac{1}{M}=\frac{\left(\beta-\varphi_{1}\right)}{K_{s}}+\frac{\varphi_{1}}{K_{w}}
$$

The effective bulk modulus, $K$, can be calculated as follows:

$$
K=K_{s}(1-\beta)+\beta^{2} M
$$

where the $K_{S}$ (and $G_{S}$ in Equation A46) are the HS average matrix bulk (and shear) modulus based on the individual soil matrix constituents (see Equation A4).

The effective shear modulus, $G$, can be estimated by assuming the $V_{s} / V_{p}$ ratio of the sediment is the multiplication of the porosity and the $V_{S} / V_{p}$ ratio of the matrix (expressed by $K_{S}$ and $G_{S}$ according to Equation A4):

$$
G=\frac{G_{S} K_{S}(1-\beta)\left(1-\varphi_{1}\right)^{2}+G_{S} \beta^{2} M\left(1-\varphi_{1}\right)^{2}}{K_{S}+4 G_{S}\left[1-\left(1-\varphi_{1}\right)^{2}\right] / 3}
$$

\section{Data Availability Statement}

Data used in this study are available from the original publications: Nakano et al. (1972), Nakano and Arnold (1973), King et al. (1982), Zimmerman and King (1986), Deschatres et al. (1988), Thimus et al. (1991), Wang et al. (2006), Christ and Park (2009), Park and Lee (2014), Dou et al. (2016), and Matsushima et al. (2016).

\section{Acknowledgments}

This publication is part of the Nunataryuk project. The project has received funding under the European Union's Horizon 2020 Research and Innovation Programme under Grant Agreement 773421. It is also supported by the Research Council of Norway through its Centers of Excellence funding Scheme, PoreLab, Project 262644. We are also grateful for valuable comments from two anonymous reviewers and Gudmund Reidar Eiksund, professor at the Norwegian University of Science and Technology.

\section{References}

Anderson, D., \& Tice, A. R. (1973). The unfrozen interfacial phase in frozen soil water systems. Physical aspects of soil water and salts in ecosystems, 107-124. Springer

Avseth, P., Mukerji, T., Mavko, G., \& Dvorkin, J. (2010). Rock-physics diagnostics of depositional texture, diagenetic alterations, and reservoir heterogeneity in high-porosity siliciclastic sediments and rocks-A review of selected models and suggested work flows. Geophysics, 75(5), 75A31-75A47. https://doi.org/10.1190/1.3483770

Biot, M. A. (1956a). Theory of propagation of elastic waves in a fluid-saturated porous solid. I. Low-Frequency Range. The Journal of the Acoustical Society of America, 28(2), 168-178. https://doi.org/10.1121/1.1908239

Biot, M. A. (1956b). Theory of propagation of elastic waves in a fluid-saturated porous solid. II. Higher Frequency Range. The Journal of the Acoustical Society of America, 28(2), 179-191. https://doi.org/10.1121/1.1908241

Bittelli, M., Flury, M., \& Campbell, G. S. (2003). A thermodielectric analyzer to measure the freezing and moisture characteristic of porous media. Water Resources Research, 39(2). https://doi.org/10.1029/2001WR000930

Carcione, J. M., Morency, C., \& Santos, J. E. (2010). Computational poroelasticity-A review. Geophysics, 75(5), 75A229-275A243. https:// doi.org/10.1190/1.3474602

Carcione, J. M., \& Seriani, G. (1998, Jul). Seismic and ultrasonic velocities in permafrost. Geophysical Prospecting, 46(4), 441-454. https:// doi.org/10.1046/j.1365-2478.1998.1000333.x

Carcione, J. M., \& Tinivella, U. (2000). Bottom-simulating reflectors: Seismic velocities and AVO effects. Geophysics, 65(1), 54-67. https:// doi.org/10.1190/1.1444725

Carns, R. C., Brandt, R. E., \& Warren, S. G. (2015). Salt precipitation in sea ice and its effect on albedo, with application to Snowball Earth. Journal of Geophysical Research: Oceans, 120(11), 7400-7412. https://doi.org/10.1002/2015JC011119

Castagna, J. P., Batzle, M. L., \& Eastwood, R. L. (1985). Relationships between compressional-wave and shear-wave velocities in clastic silicate rocks. Geophysics, 50(4), 571-581. https://doi.org/10.1190/1.1441933

Chand, S., Minshull, T. A., Gei, D., \& Carcione, J. M. (2004). Elastic velocity models for gas-hydrate-bearing sediments-A comparison. Geophysical Journal International, 159(2), 573-590. https://doi.org/10.1111/j.1365-246X.2004.02387.x

Christ, M., \& Park, J.-B. (2009). Ultrasonic technique as tool for determining physical and mechanical properties of frozen soils. Cold Regions Science and Technology, 58(3), 136-142. https://doi.org/10.1016/j.coldregions.2009.05.008

Deschatres, M., Cohen-Tenoudji, F., Aguirre-Puente, J., \& Khastou, B. (1988). Acoustic and unfrozen water content determination. Proc. 5th Inl. Conf. on permafrost.

Dillon, H. B., \& Andersland, O. B. (1966). Predicting unfrozen water contents in frozen soils. Canadian Geotechnical Journal, 3(2), 53-60. https://doi.org/10.1139/t66-007

Domenico, S. N. (1984). Rock lithology and porosity determination from shear and compressional wave velocity. Geophysics, 49(8), 1188-1195. https://doi.org/10.1190/1.1441748 
Dou, S., Nakagawa, S., Dreger, D., \& Ajo-Franklin, J. (2016). A rock-physics investigation of unconsolidated saline permafrost: $P$-wave properties from laboratory ultrasonic measurements. Geophysics, 81(1), Wa233-Wa245. https://doi.org/10.1190/Geo2015-0176.1

Dou, S., Nakagawa, S., Dreger, D., \& Ajo-Franklin, J. (2017). An effective-medium model for $P$-wave velocities of saturated, unconsolidated saline permafrost. Geophysics, 82(3), En33-En50. https://doi.org/10.1190/Geo2016-0474.1

Dvorkin, J., Berryman, J., \& Nur, A. (1999). Elastic moduli of cemented sphere packs. Mechanics of Materials, 31(7), 461-469. https://doi. org/10.1016/S0167-6636(99)00009-5

Dvorkin, J., \& Nur, A. (1996). Elasticity of high-porosity sandstones: Theory for two North Sea data sets. Geophysics, 61(5), 1363-1370. https://doi.org/10.1190/1.1444059

Dvorkin, J., Nur, A., \& Yin, H. Z. (1994). Effective properties of cemented granular-materials. Mechanics of Materials, 18(4), 351-366. https://doi.org/10.1016/0167-6636(94)90044-2

Ecker, C., Dvorkin, J., \& Nur, A. (1998). Sediments with gas hydrates: Internal structure from seismic AVO. Geophysics, 63(5), 1659-1669. https://doi.org/10.1190/1.1444462

Gei, D., \& Carcione, J. M. (2003). Acoustic properties of sediments saturated with gas hydrate, free gas and water. Geophysical Prospecting, 51(2), 141-158. https://doi.org/10.1046/j.1365-2478.2003.00359.x

Guerin, G., Goldberg, D., \& Meltser, A. (1999). Characterization of in situ elastic properties of gas hydrate-bearing sediments on the Blake Ridge. Journal of Geophysical Research: Solid Earth, 104(B8), 17781-17795. https://doi.org/10.1029/1999JB900127

Harrysson Drotz, S., Tilston, E. L., Sparrman, T., Schleucher, J., Nilsson, M., \& Öquist, M. G. (2009). Contributions of matric and osmotic potentials to the unfrozen water content of frozen soils. Geoderma, 148(3-4), 392-398. https://doi.org/10.1016/j.geoderma. 2008.11.007

Hauck, C., Böttcher, M., \& Maurer, H. (2011). A new model for estimating subsurface ice content based on combined electrical and seismic data sets. The Cryosphere, 5(2), 453-468. https://doi.org/10.5194/tc-5-453-2011

Hauck, C., Vieira, G., Gruber, S., Blanco, J., \& Ramos, M. (2007). Geophysical identification of permafrost in Livingston Island, maritime Antarctica. Journal of Geophysical Research, 112(F2), F02S19. https://doi.org/10.1029/2006JF000544

Helgerud, M. B., Dvorkin, J., Nur, A., Sakai, A., \& Collett, T. (1999). Elastic-wave velocity in marine sediments with gas hydrates: Effective medium modeling. Geophysical Research Letters, 26(13), 2021-2024. https://doi.org/10.1029/1999GL900421

Hill, R. (1952). The elastic behaviour of a crystalline aggregate. Proceedings of the Physical Society. Section A, 65(5), 349. https://doi.org/ $10.1088 / 0370-1298 / 65 / 5 / 307$

Horiguchi, K. (1987). An osmotic model for soil freezing. Cold Regions Science and Technology, 14(1), 13-22. https://doi.org/10.1016/0165232x(87)90040-1

Hu, G. W., Ye, Y. G., Zhang, J., Liu, C. L., Diao, S. B., \& Wang, J. S. (2010). Acoustic properties of gas hydrate-bearing consolidated sediments and experimental testing of elastic velocity models. Journal of Geophysical Research, 115(B2), B02102. https://doi.org/10.1029/ 2008JB006160

Humlum, O., Instanes, A., \& Sollid, J. L. (2003). Permafrost in Svalbard: A review of research history, climatic background and engineering challenges. Polar Research, 22(2), 191-215. https://doi.org/10.1111/j.1751-8369.2003.tb00107.x

Kim, H. S., Riedel, M., Ryu, B. J., Kim, G. Y., \& Bahk, J. J. (2013). Improving gas hydrate saturation estimates using $P$-wave velocity log data by incorporating XRD-data for detailed matrix-mineralogy definition. Marine and Petroleum Geology, 47, 155-167. https://doi.org/ 10.1016/j.marpetgeo.2013.05.020

King, M. (1984). The influence of clay-sized particles on seismic velocity for Canadian Arctic permafrost. Canadian Journal of Earth Sciences, 21(1), 19-24. https://doi.org/10.1139/e84-003

King, M., Pandit, B., Hunter, J., \& Gajtani, M. (1982). Some seismic, electrical, and thermal properties of sub-seabottom permafrost from the Beaufort Sea. Proceedings of the fourth Canadian permafrost conference. Ottawa: Associate Committee on Geotechnical Research, National Research Council of Canada.

King, M. S., Zimmerman, R. W., \& Corwin, R. F. (1988). Seismic and electrical-properties of unconsolidated permafrost. Geophysical Prospecting, 36(4), 349-364. https://doi.org/10.1111/j.1365-2478.1988.tb02168.x

Kleinberg, R., \& Dai, J. (2005). Estimation of the mechanical properties of natural gas hydrate deposits from petrophysical measurements. Houston, Texas: Offshore Technology Conference.

Kneisel, C., Hauck, C., Fortier, R., \& Moorman, B. (2008). Advances in geophysical methods for permafrost investigations. Permafrost and Periglacial Processes, 19(2), 157-178. https://doi.org/10.1002/ppp.616

Konno, Y., Jin, Y., Yoneda, J., Kida, M., Egawa, K., Ito, T., et al. (2015). Effect of methane hydrate morphology on compressional wave velocity of sandy sediments: Analysis of pressure cores obtained in the Eastern Nankai Trough. Marine and Petroleum Geology, 66, 425-433. https://doi.org/10.1016/j.marpetgeo.2015.02.021

Koven, C. D., Riley, W. J., \& Stern, A. (2013). Analysis of permafrost thermal dynamics and response to climate change in the CMIP5 Earth System Models. Journal of Climate, 26(6), 1877-1900. https://doi.org/10.1175/JCLI-D-12-00228.1

Koven, C. D., Ringeval, B., Friedlingstein, P., Ciais, P., Cadule, P., Khvorostyanov, D., et al. (2011). Permafrost carbon-climate feedbacks accelerate global warming. Proceedings of the National Academy of Sciences of the United States of America, 108(36), 14769-14774. https://doi.org/10.1073/pnas.1103910108

Kozlowski, T. (2009). Some factors affecting supercooling and the equilibrium freezing point in soil-water systems. Cold Regions Science and Technology, 59(1), 25-33. https://doi.org/10.1016/j.coldregions.2009.05.009

Kozlowski, T., \& Nartowska, E. (2013). Unfrozen water content in representative bentonites of different origin subjected to cyclic freezing and thawing. Vadose Zone Journal, 12(1), vzj2012.0057. https://doi.org/10.2136/vzj2012.0057

Kruse, A. M., \& Darrow, M. M. (2017). Adsorbed cation effects on unfrozen water in fine-grained frozen soil measured using pulsed nuclear magnetic resonance. Cold Regions Science and Technology, 142, 42-54. https://doi.org/10.1016/j.coldregions.2017.07.006

Kruse, A. M., Darrow, M. M., \& Akagawa, S. (2018). Improvements in measuring unfrozen water in frozen soils using the pulsed nuclear magnetic resonance method. Journal of Cold Regions Engineering, 32(1), 04017016. https://doi.org/10.1061/(ASCE)CR.1943-5495. 0000141

Kuster, G. T., \& Toksöz, M. N. (1974). Velocity and attenuation of seismic waves in two-phase media: Part I. Theoretical formulations. Geophysics, 39(5), 587-606. https://doi.org/10.1190/1.1440450

Leclaire, P., Cohen-Ténoudji, F., \& Aguirre-Puente, J. (1994). Extension of Biot's theory of wave propagation to frozen porous media. The Journal of the Acoustical Society of America, 96(6), 3753-3768. https://doi.org/10.1121/1.411336

Lee, M. W. (2002). Biot-Gassmann theory for velocities of gas hydrate-bearing sediments. Geophysics, 67(6), 1711-1719. https://doi.org/ $10.1190 / 1.1527072$ 
Lee, M. W., Hutchinson, D. R., Collett, T. S., \& Dillon, W. P. (1996). Seismic velocities for hydrate-bearing sediments using weighted equation. Journal of Geophysical Research: Solid Earth, 101(B9), 20347-20358. https://doi.org/10.1029/96JB01886

Lee, M. W., \& Waite, W. F. (2008). Estimating pore-space gas hydrate saturations from well log acoustic data. Geochemistry, Geophysics, Geosystems, 9, n/a. https://doi.org/10.1029/2008GC002081

Li, H. (2009). Experimental and numerical study of sonic wave propagation in freezing sand and silt http://hdl.handle.net/11122/8987

Ma, T., Wei, C., Xia, X., Zhou, J., \& Chen, P. (2017). Soil freezing and soil water retention characteristics: Connection and solute effects. Journal of Performance of Constructed Facilities, 31(1). https://doi.org/10.1061/(ASCE)CF.1943-5509.0000851

Matsushima, J., Suzuki, M., Kato, Y., \& Rokugawa, S. (2016, Mar-Apr). Ultrasonic measurements of attenuation and velocity of compressional and shear waves in partially frozen unconsolidated sediment and synthetic porous rock. Geophysics, 81(2), D141-D153. https://doi.org/10.1190/Geo2015-0350.1

Minshull, T. A., Singh, S. C., \& Westbrook, G. K. (1994). Seismic velocity structure at a gas hydrate reflector, offshore western Colombia, from full waveform inversion. Journal of Geophysical Research: Solid Earth, 99(B3), 4715-4734. https://doi.org/10.1029/93JB03282

Nakano, Y., \& Arnold, R. (1973). Acoustic properties of frozen Ottawa sand. Water Resources Research, 9(1), 178-184. https://doi.org/ 10.1029/WR009i001p00178

Nakano, Y., Martin, R. J. III, \& Smith, M. (1972). Ultrasonic velocities of the dilatational and shear waves in frozen soils. Water Resources Research, 8(4), 1024-1030. https://doi.org/10.1029/WR008i004p01024

Nguyen, X.-P., Cui, Y.-J., Tang, A. M., Deng, Y., Li, X.-L., \& Wouters, L. (2013). Effects of pore water chemical composition on the hydro-mechanical behavior of natural stiff clays. Engineering Geology, 166, 52-64. https://doi.org/10.1016/j.enggeo.2013.08.009

Pan, H., Li, H., Grana, D., Zhang, Y., Liu, T., \& Geng, C. (2019). Quantitative characterization of gas hydrate bearing sediment using elastic-electrical rock physics models. Marine and Petroleum Geology, 105, 273-283. https://doi.org/10.1016/j.marpetgeo.2019.04.034

Park, J.-H., \& Lee, J.-S. (2014). Characteristics of elastic waves in sand-silt mixtures due to freezing. Cold Regions Science and Technology, 99, 1-11. https://doi.org/10.1016/j.coldregions.2013.11.002

Qingbai, W., Yongzhi, L., Jianming, Z., \& Changiiang, T. (2002). A review of recent frozen soil engineering in permafrost regions along Qinghai-Tibet Highway, China. Permafrost and Periglacial Processes, 13(3), 199-205. https://doi.org/10.1002/ppp.420

Rowell, D., \& Dillon, P. (1972). Migration and aggregation of Na and Ca clays by the freezing of dispersed and flocculated suspensions. Journal of Soil Science, 23(4), 442-447. https://doi.org/10.1111/j.1365-2389.1972.tb01675.x

Santamarina, J. C., Klein, K. A., \& Fam, M. A. (2001). Soils and waves. United States: Wiley.

Schindler, M., Batzle, M. L., \& Prasad, M. (2017). Micro X-ray computed tomography imaging and ultrasonic velocity measurements in tetrahydrofuran-hydrate-bearing sediments. Geophysical Prospecting, 65(4), 1025-1036. https://doi.org/10.1111/1365-2478.12449

Smith, M. W., \& Tice, A. R. (1988). Measurement of the unfrozen water content of soils. Comparison of NMR (nuclear magnetic resonance) and TDR (time domain reflectometry) methods (no. CRREL-88-18).

Thimus, J. F., Aguirre-Puente, J., \& Cohen-Tenoudji, F. (1991). Determination of unfrozen water content of an overconsolidated clay down to $-160^{\circ} \mathrm{C}$ by sonic approaches-Comparison with classical methods. Ground freezing 91 (pp. 83-88). Rotterdam: Balkema.

Tian, H., Wei, C., Lai, Y., \& Chen, P. (2018). Quantification of water content during freeze-thaw cycles: A nuclear magnetic resonance based method. Vadose Zone Journal, 17(1), 160124. https://doi.org/10.2136/vzj2016.12.0124

Tian, H., Wei, C., Wei, H., \& Zhou, J. (2014). Freezing and thawing characteristics of frozen soils: Bound water content and hysteresis phenomenon. Cold Regions Science and Technology, 103, 74-81. https://doi.org/10.1016/j.coldregions.2014.03.007

Tice, A. R., Black, P. B., \& Berg, R. L. (1989). Unfrozen water contents of undisturbed and remolded Alaskan silt. Cold Regions Science and Technology, 17(2), 103-111. https://doi.org/10.1016/S0165-232x(89)80001-1

Timur, A. (1968). Velocity of compressional waves in porous media at permafrost temperatures. Geophysics, 33(4), 584. https://doi.org/ $10.1190 / 1.1439954$

Torrance, J. K., \& Schellekens, F. J. (2006). Chemical factors in soil freezing and frost heave. Polar Record, 42(01), 33. https://doi.org/ $10.1017 / \mathrm{s} 0032247405004894$

Vonder Mühll, D., Hauck, C., \& Gubler, H. (2002). Mapping of mountain permafrost using geophysical methods. Progress in Physical Geography, 26(4), 643-660. https://doi.org/10.1191/0309133302pp356ra

Waite, W. F., Santamarina, J. C., Cortes, D. D., Dugan, B., Espinoza, D. N., Germaine, J., et al. (2009). Physical properties of hydrate-bearing sediments. Reviews of Geophysics, 47, RG4003. https://doi.org/10.1029/2008RG000279

Wang, C., Lai, Y., Yu, F., \& Li, S. (2018). Estimating the freezing-thawing hysteresis of chloride saline soils based on the phase transition theory. Applied Thermal Engineering, 135, 22-33. https://doi.org/10.1016/j.applthermaleng.2018.02.039

Wang, C., Lai, Y., \& Zhang, M. (2017). Estimating soil freezing characteristic curve based on pore-size distribution. Applied Thermal Engineering, 124, 1049-1060. https://doi.org/10.1016/j.applthermaleng.2017.06.006

Wang, D.-y., Zhu, Y.-l., Ma, W., \& Niu, Y.-h. (2006). Application of ultrasonic technology for physical-mechanical properties of frozen soils. Cold Regions Science and Technology, 44(1), 12-19. https://doi.org/10.1016/j.coldregions.2005.06.003

Watanabe, K., \& Wake, T. (2009). Measurement of unfrozen water content and relative permittivity of frozen unsaturated soil using NMR and TDR. Cold Regions Science and Technology, 59(1), 34-41. https://doi.org/10.1016/j.coldregions.2009.05.011

Wettlaufer, J., \& Worster, M. G. (2006). Premelting dynamics. Annual Review of Fluid Mechanics, 38(1), 427-452. https://doi.org/10.1146/ annurev.fluid.37.061903.175758

Williams, P., \& Burt, T. (1974). Measurement of hydraulic conductivity of frozen soils. Canadian Geotechnical Journal, 11(4), 647-650. https://doi.org/10.1139/t74-066

Williams, P. J. (1964). Unfrozen water content of frozen soils and soil moisture suction. Geotechnique, 14(3), 231-246. https://doi.org/ 10.1680/geot.1964.14.3.231

Wyllie, M., Gregory, A., \& Gardner, G. (1958). An experimental investigation of factors affecting elastic wave velocities in porous media. Geophysics, 23(3), 459-493. https://doi.org/10.1190/1.1438493

Yun, T. S., Santamarina, J. C., \& Ruppel, C. (2007). Mechanical properties of sand, silt, and clay containing tetrahydrofuran hydrate. Journal of Geophysical Research: Solid Earth, 112. https://doi.org/10.1029/2006JB004484

Zimmerman, R. W., \& King, M. S. (1986). The effect of the extent of freezing on seismic velocities in unconsolidated permafrost. Geophysics, 51(6), 1285-1290. https://doi.org/10.1190/1.1442181 